\title{
Synthesis and Characterization of Light-Emitting Oligo( $p$-phenylene-vinylene)s and Polymeric Derivatives Containing Three- and Five-Conjugated Phenylene Rings
}

\author{
HONG-CHEU LIN, CHIEN-MIN TSAI, GUAN-HAO HUANG, JYH-MING LIN \\ Department of Materials Science and Engineering, National Chiao Tung University, \\ Hsinchu, Taiwan, Republic of China
}

Received 5 July 2005; accepted 4 October 2005

DOI: 10.1002 / pola.21173

Published online in Wiley InterScience (www.interscience.wiley.com).

\begin{abstract}
Several series of light-emitting oligo( $p$-phenylene-vinylene)s (BIII and BV series containing three- and five-conjugated phenylene rings) with various side groups and end groups attached to the cores were synthesized and characterized. The analogous PBV polymers, derived from the BV series, were also synthesized and investigated. Blue and greenish light emissions were observed in the photoluminescence (PL) and electroluminescence (EL) spectra of the blend and pure films with these $\pi$-conjugated structures. In contrast to the three-conjugated ring oligomers, the five-conjugated ring derivatives (oligomers and polymers) had larger maximum emission wavelength values of PL and EL emissions. Mesomorphism was introduced into the BV series by the replacement of three-conjugated rings (BIII series) with five-conjugated phenyl cores (BV series). The liquid-crystalline properties of the BV series with end groups (on both end rings) were better than those of analogous $\mathrm{BV}^{-\mathrm{OC}_{8}}$ without end groups. Polarized PL emissions were obtained by the alignment of liquid-crystalline phase in rubbing cells. Upon heating, different PL emission wavelengths and intensities were observed in various phases. Not only the solubility and thermal properties but also the PL and EL properties could be effectively adjusted by the attachment of flexible alkoxy groups either on the central rings or on both end rings of the conjugated cores. () 2005 Wiley Periodicals, Inc. J Polym Sci Part A: Polym Chem 44: 783-800, 2006
\end{abstract}

Keywords: electroluminescence; mesomorphism; oligo( $p$-phenylene-vinylene); photoluminescence; polarized photoluminescence

\section{INTRODUCTION}

Luminescent polymers with highly extended $\pi$ conjugated systems have attracted considerable attention from both fundamental and practical points of view because various novel physical

This article includes Supplementary Material available from the authors upon request or via the Internet at http:// www.interscience.wiley.com/jpages/0887-624X/suppmat.

Correspondence to: H.-C. Lin (E-mail: linhc@cc.nctu.edu.tw)

Journal of Polymer Science: Part A: Polymer Chemistry, Vol. 44, 783-800 (2006) @2005 Wiley Periodicals, Inc. properties have been observed and also various applications have been proposed widely. Polymers with extended $\pi$ conjugation hold promise for electronic and photonic applications, such as light-emitting diodes, photovoltaics, sensors, and thin-film transistors. ${ }^{1}$ Most liquid-crystalline (LC) materials carried out on photoluminescence (PL) and electroluminescence (EL) have involved different polarized light emissions. For instance, cyanobiphenyl liquid crystals can be aligned by rubbed polyimide and thus have dichroic ratios in absorption spectra. ${ }^{2}$ Frequently used materials 
include polyacrylate, ${ }^{3-6}$ polyfluorene,${ }^{7-18}$ and their oligomer derivatives. ${ }^{19,20}$ Additionally, liquid crystals with a hexagonal mesophase to solubilize the monomer and direct its electropolymerization lead to 3,4-polyethylene-dioxythiophene (PEDOT) films, which replicate the texture and birefringence of the LC template. ${ }^{21}$ In particular, LC conducting polymers possessing mesogenic parts in their side chains ${ }^{22-25}$ or dyes doped in an LC structure $^{26,27}$ have attracted much interest for their molecular alignment, which is expected to be used in polarized emissions. Besides, mesogenic structures containing electron-transporting 1,3,4oxadiazole units have also been used to emit polarized light. ${ }^{28,29}$ Moreover, polarized organic lightemitting diodes (OLEDs) have been constructed with heptafluorene lightly doped with monodisperse conjugated oligomers for efficient emissions of green, red, and white light, and a high polarization ratio (up to 26) has been obtained. ${ }^{30}$

Several highly extended $\pi$-conjugated systems containing oligo( $p$-phenylene-vinylene) (OPV) units are used in polymeric light-emitting diode (PLED) and OLED applications. For instance, polymerizable hexacatenar mesogens ${ }^{31}$ containing a photoactive OPV core have been thermally initiated and polymerized in the absence of light to afford effective crosslinking with the retention of both the LC order and the desired emission properties. Different generations of poly(propylene imine) dendrimers modified peripherically with urea OPV having a 3,4,5-tri(dodecyloxy)phenyl mesogenic unit (OPV dendrimers) have led to LC behavior, resulting in order on a supramolecular level. ${ }^{32}$ Besides, covalently linked dyads of OPV with [60]fullerene, ${ }^{33-35}$ amphiphilic diblock copoly(ester oxide), ${ }^{36}$ ferrocencethiol, ${ }^{37}$ and bis(thioacetyl $)^{38}$ structures exhibit interesting photophysical properties, among which energy and electron-transfer reactions have been used to make photovoltaic devices. Oligomers with defined chain lengths can be considered model compounds of their corresponding polymers. Therefore, the investigation of oligomeric OPV systems has gained interest because of their reproducible properties and their well-defined structures, which are prerequisites for the exploration of the physical properties of their corresponding polymeric materials. ${ }^{39-43}$ In comparison with the extensive attention that has been devoted to PLED systems, there are limited studies about structure-property relationships, such as the correlation between the conjugation length and the light-emitting diode performance, in oligomeric systems. ${ }^{44-52}$

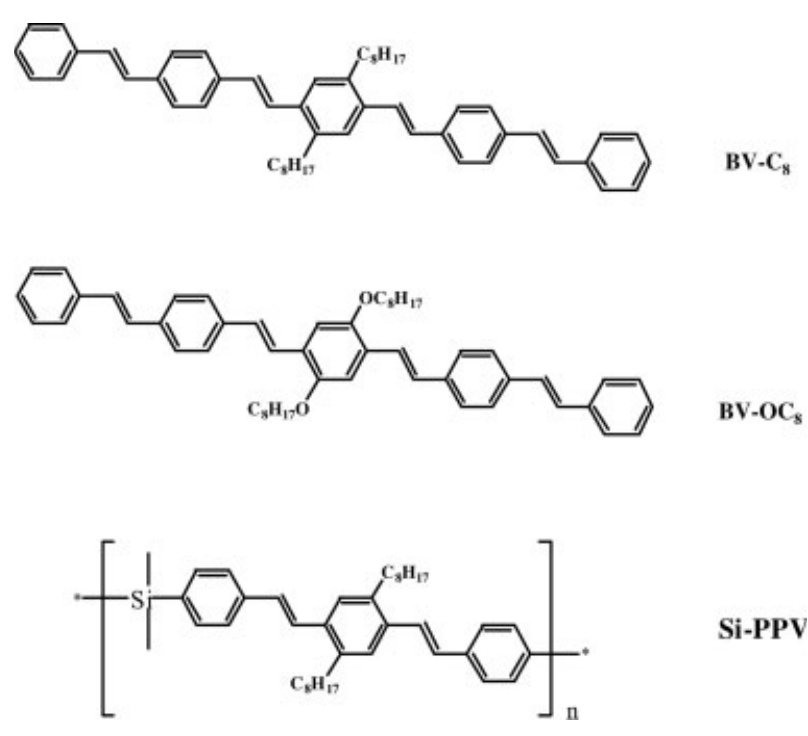

Chart 1

In some reported literature results, a series of substituted OPVs (BV-OC 8 and BV-C 8 in Chart 1) with an LC (nematic) phase were synthesized, and the emission properties were surveyed. ${ }^{53}$ In addition, a poly( $p$-phenylenevinylene) (PPV) polymer derivative [poly[2-(3-dimethyldodecylsilyphenyl)-1,4-phenylene vinylene] (Si-PPV) in Chart 1], which had a well-defined backbone consisting of regularly alternating distyrylbenzene and dimethylsilylene units, was also investigated. ${ }^{54}$ In this study, several series of alkoxysubstituted OPVs and poly( $p$-phenylene-vinylene)s containing different side groups (on the central phenyl rings) and alkoxy end groups (on both of the end phenyl rings) were explored. Various side groups, including methoxy (-OMe), butoxy $\left(-\mathrm{OC}_{4}\right)$, octoxy $\left(-\mathrm{OC}_{8}\right)$, decoxy $\left(-\mathrm{OC}_{12}\right)$, and ethylhexoxy (branched $-\mathrm{OC}_{8}$ ) side chains, were introduced into the central phenyl rings to enhance the solubility of these conjugated molecules. OPVs and analogous polymers containing three- and five-conjugated phenylene rings were synthesized to inspect the structural effects on the thermal and electrooptic properties. The rubbing effects on the PL properties, that is, the polarized emission properties, of some mesogenic compounds were also studied.

\section{EXPERIMENTAL}

\section{General Information}

NMR measurements were carried out on a Bruker AC 300 spectrometer. Absorption spectra were acquired on an HP 8453 spectrometer. PL 
and EL emission spectra were obtained on a Hitachi 8453 spectrometer. The textures of the mesophases were studied with a polarizing optical microscope (DMLP, Leica) equipped with a hot stage. The transition temperatures were determined by differential scanning calorimetry (DSC7 and Pyris 7, PerkinElmer) with a heating and cooling rate of $10{ }^{\circ} \mathrm{C} / \mathrm{min}$ under nitrogen. Thermogravimetric analysis (TGA) was conducted on a DuPont Thermal Analyst 2100 system with a TGA 2950 thermogravimetric analyzer at a heating rate of $20^{\circ} \mathrm{C} / \mathrm{min}$ under nitrogen. The highest occupied molecular orbital (HOMO) energy levels were estimated from the optical response on a Riken AC-2 photoelectron spectrometer. The work function and ionization potential were measured by the photoelectron spectrometer for the OLED materials in air. These materials were sealed in antiparallel rubbing cells with a $9-\mu \mathrm{m}$ cell gap, and the polarized PL emission spectra and PL dichroic ratios were measured at corresponding mesophasic temperatures. The textures of all mesogenic products were characterized with a Leitz Laborlux S polarizing optical microscope equipped with a THMS-600 heating stage. The heating and cooling rates were $10{ }^{\circ} \mathrm{C} \mathrm{min}{ }^{-1}$ for all measurements in $\mathrm{N}_{2}$, unless mentioned otherwise. Powder X-ray diffraction (XRD) patterns were obtained from a Siemens D-5000 X-ray diffractometer $(40 \mathrm{kV}, 30 \mathrm{~mA})$ fitted with a TTK450 temperature controller. Nickel-filtered $\mathrm{Cu} \mathrm{K} \alpha$ radiation was used as the incident $\mathrm{X}$-ray beam. The values of the number-average molecular weight $\left(M_{\mathrm{n}}\right)$, weight-average molecular weight $\left(M_{\mathrm{w}}\right)$, and polydispersity index (PDI) were determined by gel permeation chromatography (GPC). GPC analysis was conducted on a Water 1515 separation module with polystyrene as a standard and tetrahydrofuran (THF) as an eluant. The fluorescence quantum yields of solutions and films (ca. $50 \mathrm{~nm}$ ) were determined by the comparison of a reference (quinine sulfate in a $0.1 \mathrm{M}$ $\mathrm{H}_{2} \mathrm{SO}_{4}$ solution) with a known quantum yield of 0.54 (concentration $=1 \times 10^{-5} \mathrm{M}$ ) and by the comparison of poly[2-methoxy-5-(2'-ethyl-hexyloxy)1,4-phenylene] (MEHPPV) films (by the spin coating of chloroform solutions with a concentration of $1 \times 10^{-5} \mathrm{M}$ ) with a known quantum yield of 0.22 , respectively. ${ }^{7}$

\section{Device Fabrication}

Prepatterned indium tin oxide (ITO) substrates with an effective individual device area of $3.14 \mathrm{~mm}^{2}$ were cleaned by an ultrasonic machine in various detergent solutions and deionized water. After that, the ITO substrates were treated with oxygen plasma for 2 min before spin coating. Polymer thin films were spin-coated on ITO substrates from a dichloroethane solution with a concentration of $1.65 \mathrm{wt} \%$ (the thickness was ca. $60 \mathrm{~nm}$ ), whereas the molecular emitters were doped into a poly(9-vinylcarbozol) (PVK) matrix with a concentration of 1:100 wt \%. The metal calcium was deposited as the cathode by thermal evaporation and consecutively capped with aluminum serving as a protecting layer. The current-voltage-luminescence response behavior was measured under ambient conditions with a Keithley 2400 source meter and a Newport $1835 \mathrm{C}$ optical meter equipped with an 818ST silicon photodiode.

\section{Materials \\ Syntheses of OPVs (1-14)}

Commercially available chemicals were used without further purification. Diphosphonate compounds were synthesized by a modified procedure described in ref. 55. OPVs (1-14) were synthesized by the Horner-Wittig-Emmons reaction ${ }^{56}$ according to Scheme 1 [BIII series containing three-conjugated rings (1-6)] and Scheme 2 [BV series containing five-conjugated rings (7-14)]. All products were identified as the required materials and judged to be pure by ${ }^{1} \mathrm{H}$ and ${ }^{13} \mathrm{C}$ NMR spectroscopy. Elemental analyses for $\mathrm{C}, \mathrm{H}$, and $\mathrm{N}$ were also satisfactory.

BIII-OMe (1). NaH (1.5 g, $54 \mathrm{mmol}$ ) was slowly added to a solution containing $4.56 \mathrm{~g}(43 \mathrm{mmol})$ of benzaldehyde and $8 \mathrm{~g}(18 \mathrm{mmol})$ of 2,5-bismethoxy-1,4-xylene-bis(diethyl phosphonate) in $100 \mathrm{~mL}$ of anhydrous THF at $0{ }^{\circ} \mathrm{C}$ under nitrogen. The reaction mixture was slowly warmed to room temperature and refluxed for $12 \mathrm{~h}$. Upon cooling to room temperature, the mixture was poured into $500 \mathrm{~mL}$ of water. The precipitates were collected, washed with water, and crystallized from ethanol to obtain yellow crystals.

Yield: $57.0 \% .{ }^{1} \mathrm{H}$ NMR $\left(300 \mathrm{MHz}, \mathrm{CDCl}_{3}, \delta\right)$ : $3.92\left(\mathrm{~s}, 6 \mathrm{H},-\mathrm{OCH}_{3}\right), 7.12(\mathrm{~d}, J=14.5 \mathrm{~Hz}, 2 \mathrm{H}$, $-\mathrm{CH}=\mathrm{CH}-), 7.14\left(\mathrm{~s}, 2 \mathrm{H},-\mathrm{C}_{6} \mathrm{H}_{2}-\right), 7.24-7.27$ $\left(\mathrm{m}, 2 \mathrm{H},-\mathrm{C}_{6} \mathrm{H}_{5}\right), 7.35(\mathrm{t}, J=7.6 \mathrm{~Hz}, 4 \mathrm{H}$, $-\mathrm{C}_{6} \mathrm{H}_{4}-$ ), 7.53 (t, $J=8.2 \mathrm{~Hz}, 4 \mathrm{H},-\mathrm{C}_{6} \mathrm{H}_{4}-$ ). ${ }^{13} \mathrm{C} \mathrm{NMR}\left(\mathrm{CDCl}_{3}, \delta\right): 56.36,109.19,123.25$, $126.58,127.45,128.61,128.95,151.54$. ELEM. AnAL. Calcd. for $\mathrm{C}_{24} \mathrm{H}_{22} \mathrm{O}_{2}$ : C, 84.18\%; $\mathrm{H}, 6.48 \%$. Found: C, $83.80 \%$; H, $7.00 \%$. 

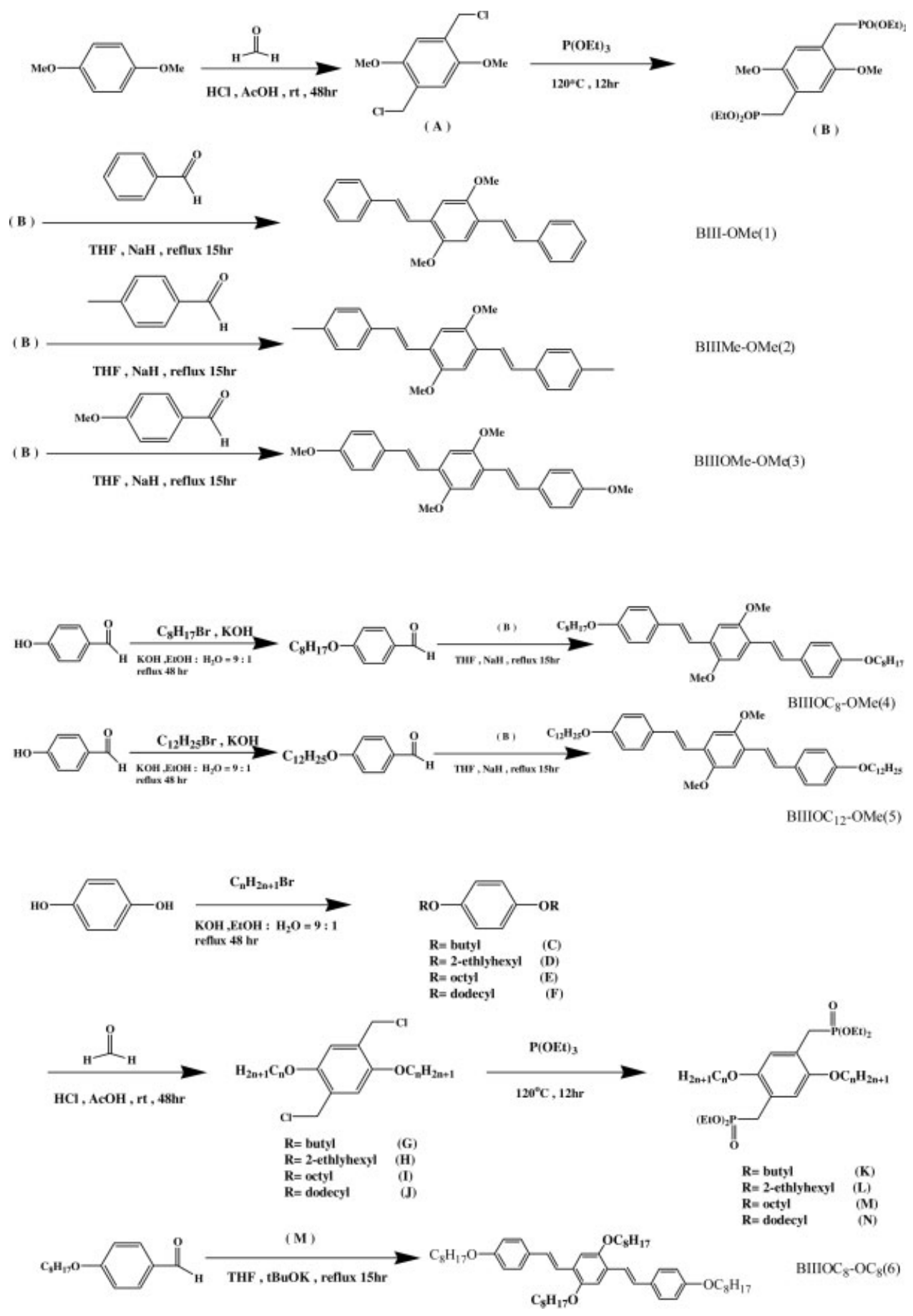

Scheme 1. Synthetic routes of OPVs containing three-conjugated rings (BIII series).

BIIIMe-OMe (2). Yield: 66\%. ${ }^{1} \mathrm{H}$ NMR (300 MHz, $\left.\mathrm{CDCl}_{3}, \delta\right): 2.36\left(\mathrm{~s}, 6 \mathrm{H},-\mathrm{CH}_{3}\right), 3.92(\mathrm{~s}, 6 \mathrm{H}$, $\left.-\mathrm{OCH}_{3}\right), 7.09(\mathrm{~d}, J=16.5 \mathrm{~Hz}, 2 \mathrm{H},-\mathrm{CH}=\mathrm{CH}-)$, $7.12\left(\mathrm{~s}, 2 \mathrm{H},-\mathrm{C}_{6} \mathrm{H}_{2}-\right), 7.16(\mathrm{~d}, J=7.8 \mathrm{~Hz}, 4 \mathrm{H}$, $\left.-\mathrm{C}_{6} \mathrm{H}_{4}-\right), 7.41(\mathrm{~d}, J=16.8 \mathrm{~Hz}, 2 \mathrm{H},-\mathrm{CH}$ $=\mathrm{CH}-), 7.44\left(\mathrm{~d}, J=7.5 \mathrm{~Hz}, 4 \mathrm{H},-\mathrm{C}_{6} \mathrm{H}_{4}-\right) .{ }^{13} \mathrm{C}$ $\mathrm{NMR}\left(\mathrm{CDCl}_{3}, \delta\right): 21.24,56.36,109.05,122.19$,
$126.47,126.55,128.76,129.32,135.05,137.30$, 151.43. Elem. AnAL. Calcd. for $\mathrm{C}_{26} \mathrm{H}_{26} \mathrm{O}_{2}$ : C, 84.29\%; H, 7.07\%. Found: C, 84.53\%; H, 7.13\%.

BIIIOMe-OMe (3). Yield: $73 \% .{ }^{1} \mathrm{H}$ NMR (300 $\left.\mathrm{MHz}, \mathrm{CDCl}_{3}, \delta\right): 3.83\left(\mathrm{~s}, 6 \mathrm{H},-\mathrm{OCH}_{3}\right), 3.92(\mathrm{~s}$, 

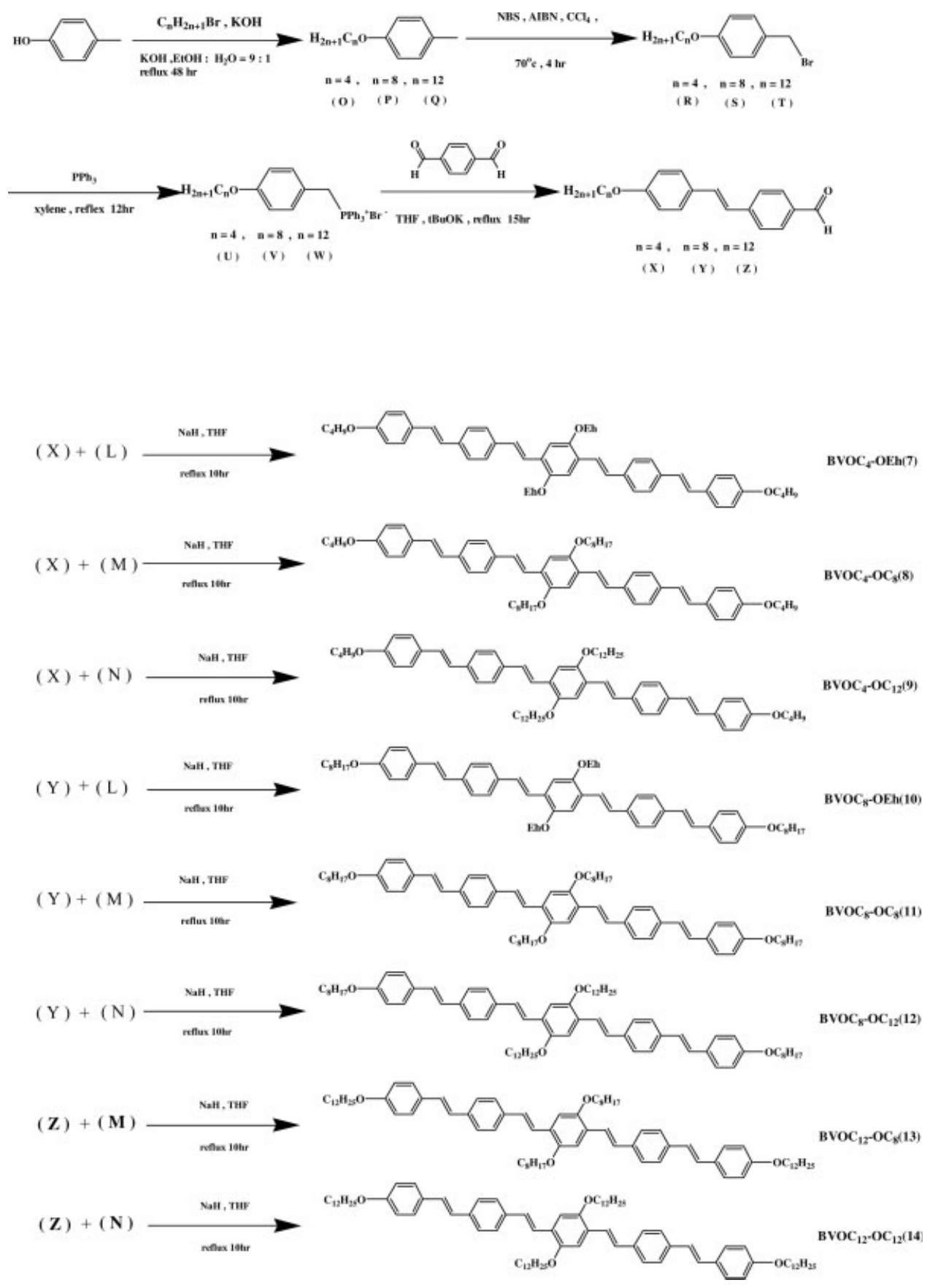

Scheme 2. Synthetic routes of OPVs containing five-conjugated rings (BV series). AIBN $=2,2$-azobis(isobutyronitrile); NBS $=$ N-bromo-succinimide.

$\left.6 \mathrm{H},-\mathrm{OCH}_{3}\right), 6.90\left(\mathrm{~d}, \mathrm{~J}=8.6 \mathrm{~Hz}, 4 \mathrm{H},-\mathrm{C}_{6} \mathrm{H}_{4}-\right.$, ), $7.06(\mathrm{~d}, \mathrm{~J}=16.4 \mathrm{~Hz}, 2 \mathrm{H},-\mathrm{CH}=\mathrm{CH}-), 7.11(\mathrm{~s}$, $\left.2 \mathrm{H},-\mathrm{C}_{6} \mathrm{H}_{2}-\right), 7.35(\mathrm{~d}, J=16.3 \mathrm{~Hz}, 2 \mathrm{H},-\mathrm{CH}$ $=\mathrm{CH}-), 7.49\left(\mathrm{~d}, 4 \mathrm{H},-\mathrm{C}_{6} \mathrm{H}_{4}-, J=8.8 \mathrm{~Hz}\right) .{ }^{13} \mathrm{C}$ NMR $\left(\mathrm{CDCl}_{3}, \delta\right)$ : 55.37, 56.37, 108.92, 114.07, 121.15 , 126.52, 127.77, 128.30, 130.72, 151.35, 159.18. Elem. Anal. Calcd. for $\mathrm{C}_{26} \mathrm{H}_{26} \mathrm{O}_{4}: \mathrm{C}$, 77.59\%; H, 6.51\%. Found: C, 77.57\%; H,6.49\%.
BIIIOC $_{8^{-}} \mathrm{OMe}$ (4). Yield: $70 \% .{ }^{1} \mathrm{H}$ NMR (300 $\left.\mathrm{MHz} \mathrm{CDCl}_{3}, \delta\right): 0.90\left(\mathrm{t}, 6 \mathrm{H},-\mathrm{CH}_{3}\right), 1.30-1.46$ $\left[\mathrm{m}, 20 \mathrm{H},-\left(\mathrm{CH}_{2}\right)_{5}-\right], 1.79\left(\mathrm{~m}, 4 \mathrm{H},-\mathrm{CH}_{2}-\right)$, $3.92\left(\mathrm{~s}, 6 \mathrm{H},-\mathrm{OCH}_{3}\right), 3.97(\mathrm{t}, J=6.9 \mathrm{~Hz}, 4 \mathrm{H}$, $\left.-\mathrm{OCH}_{2}-\right), 6.89\left(\mathrm{~d}, J=8.4 \mathrm{~Hz}, 4 \mathrm{H},-\mathrm{C}_{6} \mathrm{H}_{4}-\right)$, $7.06(\mathrm{~d}, J=16.2 \mathrm{~Hz}, 2 \mathrm{H},-\mathrm{CH}=\mathrm{CH}-), 7.11(\mathrm{~s}$, $\left.2 \mathrm{H},-\mathrm{C}_{6} \mathrm{H}_{2}-\right), 7.34(\mathrm{~d}, J=16.2 \mathrm{~Hz}, 2 \mathrm{H}$, $-\mathrm{CH}=\mathrm{CH}-), \quad 7.48(\mathrm{~d}, \quad J=8.7 \mathrm{~Hz}, 4 \mathrm{H}$, 
$\left.-\mathrm{C}_{6} \mathrm{H}_{4}-\right) .{ }^{13} \mathrm{C} \mathrm{NMR}\left(\mathrm{CDCl}_{3}, \delta\right): 14.09,22.65$, 26.05, 29.28, 29.36, 31.81, 56.38, 68.07, 108.91, $114.65,120.96,126.52,127.73,128.36,130.49$, 151.33. Elem. Anal. Calcd. for $\mathrm{C}_{40} \mathrm{H}_{54} \mathrm{O}_{4}$ : C, 80.22\%; $\mathrm{H}, 9.09 \%$. Found: C, $79.96 \%$; H, $8.98 \%$.

BIIIOC $_{12}-\mathrm{OMe}$ (5). Yield: $65 \% .{ }^{1} \mathrm{H}$ NMR (300 $\left.\mathrm{MHz} \mathrm{CDCl}_{3}, \delta\right): 0.89$ (t, $\left.6 \mathrm{H},-\mathrm{CH}_{3}\right), 1.27-1.54[\mathrm{~m}$, $36 \mathrm{H},-\left(\mathrm{CH}_{2}\right)_{9}-\mathrm{]}, 1.78\left(\mathrm{~m}, 4 \mathrm{H},-\mathrm{CH}_{2}-\right), 3.92(\mathrm{~s}$, $6 \mathrm{H},-\mathrm{OCH}_{3}$ ), $3.97\left(\mathrm{t}, J=6.6 \mathrm{~Hz}, 4 \mathrm{H},-\mathrm{OCH}_{2}-\right.$ ), $6.89\left(\mathrm{~d}, J=9.0 \mathrm{~Hz}, 4 \mathrm{H},-\mathrm{C}_{6} \mathrm{H}_{4}-\right), 7.06(\mathrm{~d}, J$ $=16.4 \mathrm{~Hz}, 2 \mathrm{H},-\mathrm{CH}=\mathrm{CH}-), 7.11(\mathrm{~s}, 2 \mathrm{H}$, $\left.-\mathrm{C}_{6} \mathrm{H}_{2}-\right), \quad 7.34(\mathrm{~d}, \quad J=16.5 \mathrm{~Hz}, 2 \mathrm{H}$, $-\mathrm{CH}=\mathrm{CH}-$ ), $7.48\left(\mathrm{~d}, J=8.7 \mathrm{~Hz}, 4 \mathrm{H},-\mathrm{C}_{6} \mathrm{H}_{4}-\right)$. ${ }^{13} \mathrm{C}$ NMR $\left(\mathrm{CDCl}_{3}, \delta\right): 14.10,22.69,26.05,29.34$, $29.41,29.59,29.63,31.91,56.40,68.09,108.98$, $114.68,121.00,126.57,127.73,128.39,130.52$, 158.81. Elem. Anal. Calcd. for $\mathrm{C}_{48} \mathrm{H}_{70} \mathrm{O}_{4}$ : C, $81.08 \%$; H, $9.92 \%$. Found: C, $81.3 \%$; H, $9.64 \%$.

BIIIOC $_{8}-O_{8}(\boldsymbol{6})$. Yield: $15 \% .{ }^{1} \mathrm{H}$ NMR $(300 \mathrm{MHz}$, $\left.\mathrm{CDCl}_{3}, \delta\right): 0.89\left(\mathrm{t}, 12 \mathrm{H},-\mathrm{CH}_{3}\right), 1.28-1.54[\mathrm{~m}$, $40 \mathrm{H},-\left(\mathrm{CH}_{2}\right)_{9}-\mathrm{]}, 1.62-1.89\left(\mathrm{~m}, 8 \mathrm{H},-\mathrm{CH}_{2}-\right)$, $3.97\left(\mathrm{t}, J=6.5 \mathrm{~Hz}, 4 \mathrm{H},-\mathrm{OCH}_{2}-\right), 4.04(\mathrm{t}, J$ $\left.=6.6 \mathrm{~Hz}, 4 \mathrm{H},-\mathrm{OCH}_{2}-\right), 6.89(\mathrm{~d}, J=8.7 \mathrm{~Hz}, 4 \mathrm{H}$, $\left.-\mathrm{C}_{6} \mathrm{H}_{4}-\right), 7.07(\mathrm{~d}, J=16.1 \mathrm{~Hz}, 2 \mathrm{H},-\mathrm{CH}=\mathrm{CH}-$ $J=6.5 \mathrm{~Hz}), 7.10\left(\mathrm{~s}, 2 \mathrm{H},-\mathrm{C}_{6} \mathrm{H}_{2}-\right), 7.33(\mathrm{~d}, J$ $=16.4 \mathrm{~Hz}, 2 \mathrm{H},-\mathrm{CH}=\mathrm{CH}-), 7.45(\mathrm{~d}, J=8.6 \mathrm{~Hz}$, $\left.4 \mathrm{H},-\mathrm{C}_{6} \mathrm{H}_{4}-\right) .{ }^{13} \mathrm{C} \mathrm{NMR}\left(\mathrm{CDCl}_{3}, \delta\right): 14.10,22.68$, 26.07, 26.31, 29.31, 29.34, 29.38, 29.41, 29.48, $29.59,29.64,29.67,31.92,110.57,114.69,121.33$, 126.89, 127.64, 128.19, 130.67, 150.97. ELEM. ANAL. Calcd. for $\mathrm{C}_{54} \mathrm{H}_{82} \mathrm{O}_{4}: \mathrm{C}, 81.56 \% ; \mathrm{H}, 10.39 \%$. Found: $\mathrm{C}, 82.51 \%$; H, $10.92 \%$.

$\mathrm{BVOC}_{4}$-OEh (7). $t$-BuOK (1.28 g, $\left.29.3 \mathrm{mmol}\right)$ was slowly added to a solution containing $6.60 \mathrm{~g}$ (19.6 mmol) of 4-butyloxy-benzyl-triphenyl-phosphonium bromide and $4.78 \mathrm{~g}(19.6 \mathrm{mmol})$ of terephthaldicarboxaldehyde in $50 \mathrm{~mL}$ of anhydrous THF at $0{ }^{\circ} \mathrm{C}$ under nitrogen. The reaction mixture was slowly warmed to $100{ }^{\circ} \mathrm{C}$ and refluxed for $20 \mathrm{~h}$. Upon cooling to room temperature, the mixture was poured into water and dichloromethane. The precipitates were collected, washed with ethyl alcohol, and crystallized from elementary analysis/hexane (1:20) to obtain yellow crystals of trans-4-(4-butyloxy)styryl benzaldehyde. Then, $2.0 \mathrm{~g}$ ( $5.9 \mathrm{mmol})$ of $\mathrm{NaH}$ was slowly added to a solution containing trans-4-(4-butyloxy)styryl benzaldehyde and $1.72 \mathrm{~g}(2.7 \mathrm{mmol})$ of $1,4-$ bis(4-formylstyryl)-2,5-bis(2-ethylhexyloxy)benzene in $50 \mathrm{~mL}$ of anhydrous $\mathrm{THF}$ at room temperature under nitrogen. The reaction mixture was slowly warmed to $100{ }^{\circ} \mathrm{C}$ and refluxed for $20 \mathrm{~h}$. Upon cooling to room temperature, the mixture was poured into water and dichloromethane. The precipitates were collected, washed with THF, and crystallized from dichloromethane to obtain golden crystals.

Yield: $25 \% .{ }^{1} \mathrm{H}$ NMR (300 $\left.\mathrm{MHz}, \mathrm{CDCl}_{3}, \delta\right)$ : 0.90-1.02 (m, 18H, $\left.-\mathrm{CH}_{3}\right), 1.36-1.56[\mathrm{~m}, 20 \mathrm{H}$, $-\left(\mathrm{CH}_{2}\right)_{n}-\mathrm{]}, 1.73-1.85\left(\mathrm{~m}, 6 \mathrm{H},-\mathrm{CH}_{2}-\right), 3.95-$ $4.00\left(\mathrm{~m}, 8 \mathrm{H},-\mathrm{OCH}_{2}-\right), 6.88(\mathrm{~d}, J=9 \mathrm{~Hz}, 4 \mathrm{H}$, $\left.-\mathrm{C}_{6} \mathrm{H}_{4}-\right), 6.94(\mathrm{~d}, J=16.2 \mathrm{~Hz}, 1 \mathrm{H},-\mathrm{CH}=\mathrm{CH}-$ ), $7.06(\mathrm{~d}, J=15 \mathrm{~Hz}, 1 \mathrm{H},-\mathrm{CH}=\mathrm{CH}-), 7.13-7.17$ (m, $6 \mathrm{H},-\mathrm{C}_{6} \mathrm{H}_{2}-,-\mathrm{CH}=\mathrm{CH}-$, overlapping), $7.43-7.53\left(\mathrm{~m}, 14 \mathrm{H},-\mathrm{C}_{6} \mathrm{H}_{4}-,-\mathrm{CH}=\mathrm{CH}-\right.$, overlapping). ${ }^{13} \mathrm{C} \mathrm{NMR}\left(\mathrm{CDCl}_{3}, \delta\right): 11.34,13.86,14.14$, $19.24,23.11,24.23,29.26,30.93,31.30,39.76$, $67.74,71.72,110.12,114.70,123.09,126.09,126.53$, $126.74,126.80,127.67,128.02,128.28,129.96$, 136.82, 136.96, 151.18, 158.89. Elem. Anal. Calcd. for $\mathrm{C}_{62} \mathrm{H}_{78} \mathrm{O}_{4}$ : C, $83.93 \%$; H, 8.86\%. Found: C, $82.48 \%$; H, $8.52 \%$.

BVOC $_{4}-\mathrm{OC}_{8}$ (8). Yield: $31 \% .{ }^{1} \mathrm{H}$ NMR $(300 \mathrm{MHz}$, $\left.\mathrm{CDCl}_{3}, \delta\right): 0.87\left(\mathrm{t}, 6 \mathrm{H},-\mathrm{CH}_{3}\right), 0.91\left(\mathrm{t}, 6 \mathrm{H},-\mathrm{CH}_{3}\right)$, 1.31-1.58 [m, 28H, - $\left.\left(\mathrm{CH}_{2}\right)_{n}-\right], 1.75-1.91(\mathrm{~m}, 8 \mathrm{H}$, $-\mathrm{CH}_{2}-$ ), $3.96\left(\mathrm{t}, J=6.6 \mathrm{~Hz}, 4 \mathrm{H},-\mathrm{OCH}_{2}-\right), 4.04$ $\left(\mathrm{t}, J=6.6 \mathrm{~Hz}, 4 \mathrm{H},-\mathrm{OCH}_{2}-\right), 6.88(\mathrm{~d}, J=8.4 \mathrm{~Hz}$, $\left.4 \mathrm{H},-\mathrm{C}_{6} \mathrm{H}_{4}-\right), 6.94(\mathrm{~d}, J=16.5 \mathrm{~Hz}, 1 \mathrm{H},-\mathrm{CH}$ $=\mathrm{CH}-), 7.05(\mathrm{~d}, J=16.2 \mathrm{~Hz}, 1 \mathrm{H},-\mathrm{CH}=\mathrm{CH}-)$, 7.10-7.15 (m, 6H, $-\mathrm{C}_{6} \mathrm{H}_{2}-,-\mathrm{CH}=\mathrm{CH}-$, overlapping), $7.43-7.52\left(\mathrm{~m}, 14 \mathrm{H},-\mathrm{C}_{6} \mathrm{H}_{4}-,-\mathrm{CH}\right.$ $=\mathrm{CH}-$, overlapping). ${ }^{13} \mathrm{C} \mathrm{NMR}\left(\mathrm{CDCl}_{3}, \delta\right): 13.86$, $14.13,19.23,22.69,26.30,29.33,29.43,29.51$, $31.30,31.83,67.73,69.57,110.51,114.69,123.09$, $126.08,126.50,126.80,126.90,127.67,128.02$, $128.38,129.94,136.83,136.91,151.10,158.88$. Elem. Anal. Calcd. for $\mathrm{C}_{62} \mathrm{H}_{78} \mathrm{O}_{4}$ : C, 83.93\%; H, 8.86\%. Found: C, $83.88 \% ; \mathrm{H}, 8.87 \%$.

BVOC $_{4}-\mathrm{OC}_{12}$ (9). Yield: $35 \% .{ }^{1} \mathrm{H} \mathrm{NMR}(300 \mathrm{MHz}$, $\left.\mathrm{CDCl}_{3}, \delta\right): 0.83\left(\mathrm{t}, 6 \mathrm{H},-\mathrm{CH}_{3}\right), 0.94\left(\mathrm{t}, 6 \mathrm{H},-\mathrm{CH}_{3}\right)$, 1.24-1.54 [m, $\left.40 \mathrm{H},-\left(\mathrm{CH}_{2}\right)_{n}-\right], 1.76-1.84(\mathrm{~m}, 8 \mathrm{H}$, $-\mathrm{CH}_{2}-$ ), $3.95\left(\mathrm{t}, J=6.6 \mathrm{~Hz}, 4 \mathrm{H},-\mathrm{OCH}_{2}-\right), 4.02$ (t, $\left.J=6.6 \mathrm{~Hz}, 4 \mathrm{H},-\mathrm{OCH}_{2}-\right), 6.86\left(\mathrm{~d}, 4 \mathrm{H},-\mathrm{C}_{6}\right.$ $\left.\mathrm{H}_{4}-, J=8.4 \mathrm{~Hz}\right), 6.92(\mathrm{~d}, J=16.2 \mathrm{~Hz}, 1 \mathrm{H},-\mathrm{CH}$ $=\mathrm{CH}-), 7.04(\mathrm{~d}, J=16.3 \mathrm{~Hz}, 1 \mathrm{H},-\mathrm{CH}=\mathrm{CH}-)$, 7.09-7.13 $\left(\mathrm{m}, 6 \mathrm{H},-\mathrm{C}_{6} \mathrm{H}_{2}-,-\mathrm{CH}=\mathrm{CH}-\right.$, overlapping), $7.41-7.50\left(\mathrm{~m}, 14 \mathrm{H},-\mathrm{C}_{6} \mathrm{H}_{4}-,-\mathrm{CH}\right.$ $=\mathrm{CH}-$, overlapping). ${ }^{13} \mathrm{C} \mathrm{NMR}\left(\mathrm{CDCl}_{3}, \delta\right): 13.85$, $14.11,19.24,22.69,26.31,29.38,29.48,29.51$, $29.65,29.67,29.73,31.32,31.93,34.11,67.76$, $69.61,110.59,114.72,123.12,126.11,126.51$, $126.81,126.95,127.67,128.04,128.41,129.98$, 136.86, 136.94, 151.13, 158.91. Elem. Anal. Calcd. 
for $\mathrm{C}_{70} \mathrm{H}_{94} \mathrm{O}_{4}$ : C, 84.12\%; $\mathrm{H}, 9.48 \%$. Found: $\mathrm{C}$, $83.59 \%$; H, $9.31 \%$.

$B_{V O C}-O E h$ (10). Yield: $30 \% .{ }^{1} \mathrm{H}$ NMR (300 $\left.\mathrm{MHz}_{\mathrm{CDCl}}, \delta\right): 0.85-0.96\left(\mathrm{~m}, 12 \mathrm{H},-\mathrm{CH}_{3}\right), 0.96$ (t, 6H, $\left.-\mathrm{CH}_{3}\right), 1.27-1.53\left[\mathrm{~m}, 36 \mathrm{H},-\left(\mathrm{CH}_{2}\right)_{n}-\right]$, 1.77-1.81 (m, 6H, $\left.-\mathrm{CH}_{2}-\right), 3.94-3.97(\mathrm{~m}, 8 \mathrm{H}$, $\left.-\mathrm{OCH}_{2}-\right), 6.86\left(\mathrm{~d}, J=8.7 \mathrm{~Hz}, 4 \mathrm{H},-\mathrm{C}_{6} \mathrm{H}_{4}-\right)$, $6.93(\mathrm{~d}, J=16.2 \mathrm{~Hz}, 1 \mathrm{H},-\mathrm{CH}=\mathrm{CH}-), 7.04(\mathrm{~d}$, $J=15 \mathrm{~Hz}, 1 \mathrm{H},-\mathrm{CH}=\mathrm{CH}-), 7.10-7.14(\mathrm{~m}, 6 \mathrm{H}$, $-\mathrm{C}_{6} \mathrm{H}_{2}-,-\mathrm{CH}=\mathrm{CH}-$, overlapping), 7.41-7.50 (m, $14 \mathrm{H},-\mathrm{C}_{6} \mathrm{H}_{4}-,-\mathrm{CH}=\mathrm{CH}-$, overlapping). ${ }^{13} \mathrm{C}$ NMR $\left(\mathrm{CDCl}_{3}, \delta\right): 11.34,14.09,14.13,22.65$, $23.11,24.26,26.04,29.23,29.27,29.36,30.95$, $31.81,39.79,68.09,71.77,110.17,114.73,123.12$, $126.11,126.53,126.75,126.84,127.67,128.05$, 128.30, 129.97, 136.85, 136.98, 151.21, 158.91 . Elem. Anal. Calcd. for $\mathrm{C}_{70} \mathrm{H}_{94} \mathrm{O}_{4}$ : C, $84.12 \%$; $\mathrm{H}$, 9.48\%. Found: C, $83.76 \%$; H, 9.07\%.

$\mathrm{BVOC}_{8^{-}} \mathrm{OC}_{8}$ (11). Yield: $45 \% .{ }^{1} \mathrm{H}$ NMR (300 MHz, $\left.\mathrm{CDCl}_{3}, \delta\right): 0.89\left(\mathrm{t}, 12 \mathrm{H},-\mathrm{CH}_{3}\right), 1.31-1.57[\mathrm{~m}, 40 \mathrm{H}$, $\left.-\left(\mathrm{CH}_{2}\right)_{n}-\right], 1.77-1.91\left(\mathrm{~m}, 8 \mathrm{H},-\mathrm{CH}_{2}-\right), 3.97(\mathrm{t}$, $\left.J=6.5 \mathrm{~Hz}, 4 \mathrm{H},-\mathrm{OCH}_{2}-\right), 4.06\left(\mathrm{t}, 4 \mathrm{H},-\mathrm{OCH}_{2}-\right.$, $J=6.5 \mathrm{~Hz}), 6.89\left(\mathrm{~d}, J=8.8 \mathrm{~Hz}, 4 \mathrm{H},-\mathrm{C}_{6} \mathrm{H}_{4}-\right)$, $6.97(\mathrm{~d}, J=16.3 \mathrm{~Hz}, 2 \mathrm{H},-\mathrm{CH}=\mathrm{CH}-), 7.05-7.16$ (m, $6 \mathrm{H},-\mathrm{C}_{6} \mathrm{H}_{2}-,-\mathrm{CH}=\mathrm{CH}-$, overlapping), 7.43-7.53 $\left(\mathrm{m}, 14 \mathrm{H},-\mathrm{C}_{6} \mathrm{H}_{4}-,-\mathrm{CH}=\mathrm{CH}-\right.$, overlapping). ${ }^{13} \mathrm{C} \mathrm{NMR}\left(\mathrm{CDCl}_{3}, \delta\right): 14.10,22.65,22.68$, $26.05,26.31,29.23,29.27,29.32,29.36,29.43$, $29.52,31.80,31.84,68.09,69.61,110.60,114.73$, $126.11,126.50,126.81,126.96,127.68,128.06$, 128.41, 129.97, 136.87, 136.94, 151.15. Elem. Anal. Calcd. for $\mathrm{C}_{70} \mathrm{H}_{94} \mathrm{O}_{4}$ : C, 84.12\%; H, 9.48\%. Found: C, $84.07 \%$; H, $9.26 \%$.

$B O C_{8}-O C_{12}$ (12). Yield: $21 \% .{ }^{1} \mathrm{H}$ NMR (300 $\mathrm{MHz}, \mathrm{CDCl}_{3}, \delta$ ): 0.87 (t, $\left.12 \mathrm{H},-\mathrm{CH}_{3}\right), 1.27-1.57$ $\left[\mathrm{m}, \quad 56 \mathrm{H},-\left(\mathrm{CH}_{2}\right)_{n}-\right], \quad 1.77-1.91(\mathrm{~m}, \quad 8 \mathrm{H}$, $-\mathrm{CH}_{2}-$ ), $3.98\left(\mathrm{t}, J=6.8 \mathrm{~Hz}, 4 \mathrm{H},-\mathrm{OCH}_{2}-\right.$ ), $4.06\left(\mathrm{t}, J=6.4 \mathrm{~Hz}, 4 \mathrm{H},-\mathrm{OCH}_{2}-\right), 6.89(\mathrm{~d}, J$ $\left.=8.5 \mathrm{~Hz}, 4 \mathrm{H},-\mathrm{C}_{6} \mathrm{H}_{4}-\right), 6.97(\mathrm{~d}, J=16.3 \mathrm{~Hz}$, $2 \mathrm{H},-\mathrm{CH}=\mathrm{CH}-), 7.06-7.16\left(\mathrm{~m}, 6 \mathrm{H},-\mathrm{C}_{6} \mathrm{H}_{2}-\right.$, $-\mathrm{CH}=\mathrm{CH}-$, overlapping), $7.43-7.53(\mathrm{~m}, 14 \mathrm{H}$, $-\mathrm{C}_{6} \mathrm{H}_{4}-,-\mathrm{CH}=\mathrm{CH}-$, overlapping). ${ }^{13} \mathrm{C} \mathrm{NMR}$ $\left(\mathrm{CDCl}_{3}, \delta\right): 6.90,14.10,22.68,26.05,26.32,29.25$, $29.38,29.49,29.68,31.82,31.93,68.12,69.64$, $110.66,114.75,123.76,123.16,126.13,126.52$, $126.82,127.68,128.07,128.43,130.00,136.96$, 151.17, 158.93. Elem. Anal. Calcd. for $\mathrm{C}_{78} \mathrm{H}_{110} \mathrm{O}_{4}$ : C, $84.27 \%$; H, 9.97\%. Found: C, 84.48\%; H, 9.71\%.

$B O C_{12}-O C_{8}$ (13). Yield: $19 \% .{ }^{1} \mathrm{H}$ NMR (300 $\mathrm{MHz}, \mathrm{CDCl}_{3}, \delta$ ):0.82 (t, $12 \mathrm{H},-\mathrm{CH}_{3}$ ), 1.20-1.50 $\left[\mathrm{m}, \quad 56 \mathrm{H},-\left(\mathrm{CH}_{2}\right)_{n}-\right], \quad 1.69-1.81 \quad(\mathrm{~m}, \quad 8 \mathrm{H}$, $-\mathrm{CH}_{2}-$ ), 3.91 (t, $J=6.7 \mathrm{~Hz}, 4 \mathrm{H},-\mathrm{OCH}_{2}-$ ), $3.99\left(\mathrm{t}, J=6.2 \mathrm{~Hz}, 4 \mathrm{H},-\mathrm{OCH}_{2}-\right), 6.82(\mathrm{~d}, J$ $\left.=8.7 \mathrm{~Hz}, 4 \mathrm{H},-\mathrm{C}_{6} \mathrm{H}_{4}-\right), 6.90(\mathrm{~d}, J=16.2 \mathrm{~Hz}$, $2 \mathrm{H},-\mathrm{CH}=\mathrm{CH}-), 6.99-7.09\left(\mathrm{~m}, 6 \mathrm{H},-\mathrm{C}_{6} \mathrm{H}_{2}-\right.$, $-\mathrm{CH}=\mathrm{CH}-$, overlapping), 7.36-7.45 (m, $14 \mathrm{H}$, $-\mathrm{C}_{6} \mathrm{H}_{4}-,-\mathrm{CH}=\mathrm{CH}-$, overlapping). ${ }^{13} \mathrm{C} \mathrm{NMR}$ $\left(\mathrm{CDCl}_{3}, \delta\right): 1.03,14.12,22.70,26.05,26.32,29.28$, $29.35,29.43,29.44,29.54,29.58,29.63,29.68$, $31.85,31.93,68.10,69.62,110.60,114.73,123.14$, $126.12,126.53,126.82,126.97,127.69,128.08$, $128.42,129.99,136.88,136.95,151.14,158.92$, 176.32. Elem. Anal. Calcd. for $\mathrm{C}_{78} \mathrm{H}_{110} \mathrm{O}_{4}$ : C, $84.27 \%$; H, 9.97\%. Found: C, $84.02 \%$; H, $9.51 \%$.

$B V O C_{12}-O C_{12}$ (14). Yield: $12 \% .{ }^{1} \mathrm{H}$ NMR (300 $\mathrm{MHz}, \mathrm{CDCl}_{3}, \delta$ ): 0.87 (t, $12 \mathrm{H},-\mathrm{CH}_{3}$ ), 1.27-1.58 $\left[\mathrm{m}, \quad 72 \mathrm{H},-\left(\mathrm{CH}_{2}\right)_{9}-\right], \quad 1.79-1.88(\mathrm{~m}, \quad 8 \mathrm{H}$, $-\mathrm{CH}_{2}-$ ), 3.98 (t, $J=6.5 \mathrm{~Hz}, 4 \mathrm{H},-\mathrm{OCH}_{2}-$ ), $4.07\left(\mathrm{t}, J=6.4 \mathrm{~Hz}, 4 \mathrm{H},-\mathrm{OCH}_{2}-\right), 6.89(\mathrm{~d}, J$ $\left.=8.7 \mathrm{~Hz}, 4 \mathrm{H},-\mathrm{C}_{6} \mathrm{H}_{4}-\right), 6.97(\mathrm{~d}, J=16.4 \mathrm{~Hz}$, $2 \mathrm{H},-\mathrm{CH}=\mathrm{CH}-), 7.06-7.16\left(\mathrm{~m}, 6 \mathrm{H},-\mathrm{C}_{6} \mathrm{H}_{2}-\right.$, $-\mathrm{CH}=\mathrm{CH}-$, overlapping), $7.48\left(\mathrm{~m}, 14 \mathrm{H},-\mathrm{C}_{6} \mathrm{H}_{4}-\right.$, $-\mathrm{CH}=\mathrm{CH}-$, overlapping). ${ }^{13} \mathrm{C} \mathrm{NMR}\left(\mathrm{CDCl}_{3}, \delta\right)$ : $14.12,22.68,29.39,29.48,29.59,29.64,29.72$, 31.93, 114.73, 126.53, 126.80, 127.67. Elem. ANAL. Calcd. for $\mathrm{C}_{86} \mathrm{H}_{126} \mathrm{O}_{4}: \mathrm{C}, 84.39 \%$; $\mathrm{H}, 10.38 \%$. Found: C, $83.87 \%$; H, $10.10 \%$.

\section{Synthesis of the Polymers (15-18)}

Poly( $p$-phenylene-vinylene)s were synthesized by the Horner-Wittig-Emmons reaction ${ }^{56}$ illustrated in Scheme 3 [PBV series containing fiveconjugated rings $(\mathbf{1 5}-\mathbf{1 8})$ ]. The synthetic protocols of polymer precursors (M1-M5) are described next. M1-M5 were identified as the required materials and judged to be pure by ${ }^{1} \mathrm{H}$ and ${ }^{13} \mathrm{C}$ NMR spectroscopy as well as elemental analyses.

1,8-Bis(4-triphenyl phosphonium bromide-phenoxy)-octane (M1). A solution of 1,8-bis(4-bromomethyl-phenoxy)-octane (8 g, $16.5 \mathrm{mmol})$ and triphenylphosphine $(9.5 \mathrm{~g}, 36.4 \mathrm{mmol})$ in $80 \mathrm{~mL}$ of xylene was heated to $120{ }^{\circ} \mathrm{C}$ for $12 \mathrm{~h}$. Upon cooling to room temperature, the reaction mixture was filtered and washed by hexane to obtain a white solid.

Yield: $84 \%$. ${ }^{1} \mathrm{H}$ NMR (300 $\left.\mathrm{MHz}, \mathrm{CDCl}_{3}, \delta\right)$ : $1.23-1.53\left[\mathrm{~m}, 8 \mathrm{H},-\left(\mathrm{CH}_{2}\right)_{4}-\right], 1.69-1.74(\mathrm{~m}, 4 \mathrm{H}$, $-\mathrm{CH}_{2}-$ ), $3.81\left(\mathrm{t}, J=6.6 \mathrm{~Hz}, 4 \mathrm{H},-\mathrm{OCH}_{2}-\right.$ ), $5.35\left(\mathrm{~d}, J=20.0 \mathrm{~Hz}, 4 \mathrm{H},-\mathrm{CH}_{2} \mathrm{PPh}_{3}{ }^{+} \mathrm{Br}^{-}\right), 6.85$ $\left(\mathrm{d}, J=8.6 \mathrm{~Hz}, 4 \mathrm{H},-\mathrm{C}_{6} \mathrm{H}_{4}-\right), 7.11(\mathrm{~d}, J=8.6 \mathrm{~Hz}$, $\left.4 \mathrm{H},-\mathrm{C}_{6} \mathrm{H}_{4}-\right), 7.53-7.74\left(\mathrm{~m}, 30 \mathrm{H}, \mathrm{PPh}_{3}\right)$. 


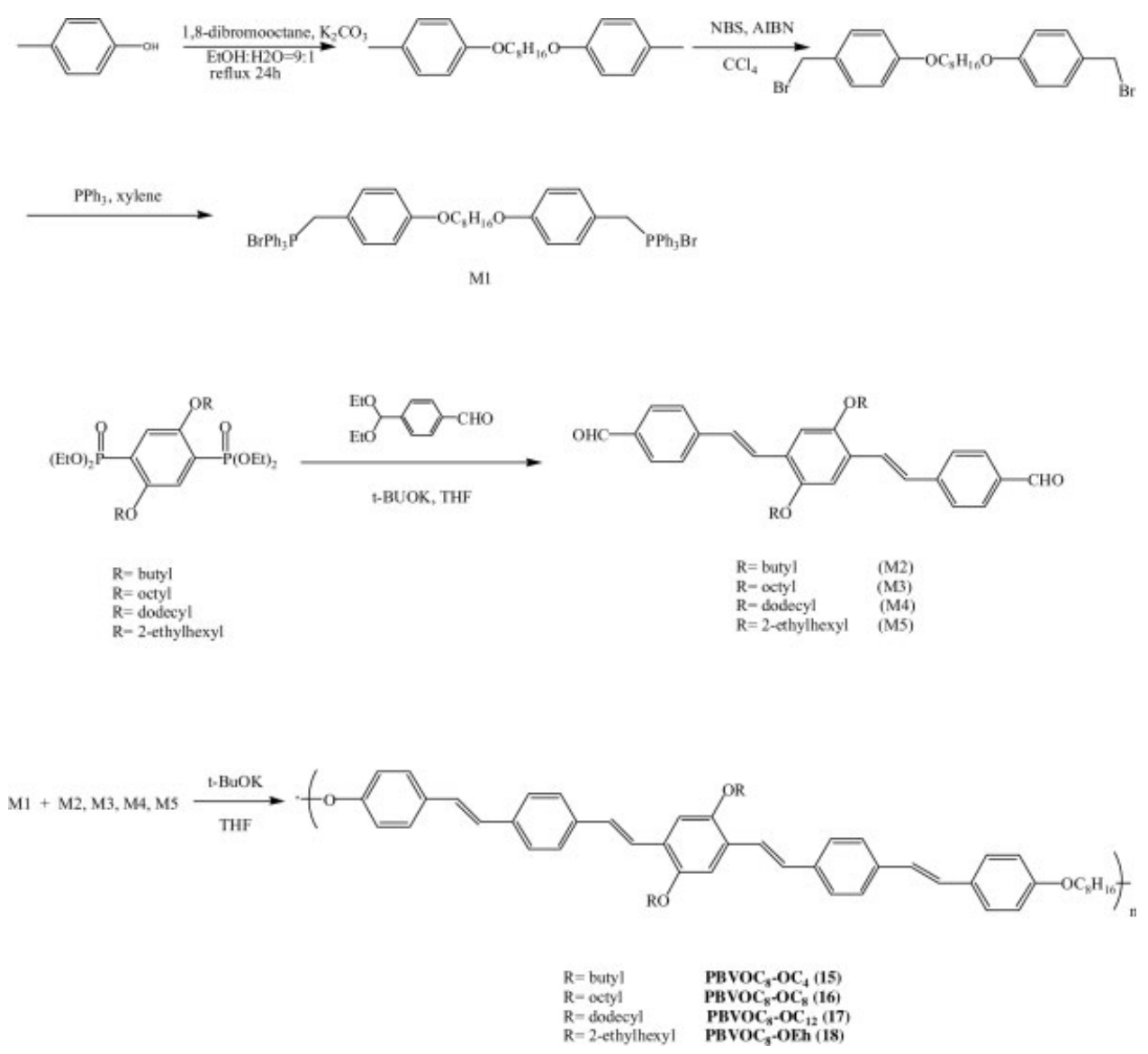

Scheme 3. Synthetic routes of poly( $p$-phenylene-vinylene)s (PBV series).

\section{1,4-Bis(4-formylstyryl)-2,5-bis(butyloxy)benzene} (M2). $t$-BuOK $(9.4 \mathrm{~g}, 100.6 \mathrm{mmol})$ was added to a solution of 2,5-bisbutyloxy-1,4-xylene-bis(diethyl phosphonate) and terephthalaldehyde-mono(diethyl acetal) (14.8 $\mathrm{mL}, 73.8 \mathrm{mmol}$ ) in anhydrous THF at $0{ }^{\circ} \mathrm{C}$. The mixture was reacted at room temperature for $30 \mathrm{~min}$ and then slowly warmed to $80{ }^{\circ} \mathrm{C}$ for another $12 \mathrm{~h}$. As the reaction mixture cooled to room temperature, $40 \mathrm{~mL}$ of $6 \mathrm{~N} \mathrm{HCl}$ was poured into the mixture to terminate the reaction. After $1 \mathrm{~h}$, the product was precipitated by the addition of $300 \mathrm{~mL}$ of cold water, and the precipitate was collected. The crude product was recrystallized from isopropyl alcohol twice to obtain an orange powder.

Yield: $49 \% .{ }^{1} \mathrm{H}$ NMR $\left(300 \mathrm{MHz}, \mathrm{CDCl}_{3}, \delta\right): 0.98$ (t, 6H, $\left.-\mathrm{CH}_{3}\right), 1.44-1.56\left[\mathrm{~m}, 4 \mathrm{H},-\left(\mathrm{CH}_{2}\right)-\right]$, $1.72-1.82\left(\mathrm{~m}, 4 \mathrm{H},-\mathrm{CH}_{2}-\right), 4.07(\mathrm{t}, J=6.6 \mathrm{~Hz}$, $\left.4 \mathrm{H},-\mathrm{OCH}_{2}-\right), 7.12\left(\mathrm{~s}, 2 \mathrm{H},-\mathrm{C}_{6} \mathrm{H}_{2}-\right), 7.15(\mathrm{~d}$, $2 \mathrm{H}, J=18 \mathrm{~Hz},-\mathrm{CH}=\mathrm{CH}-), 7.58(\mathrm{~d}, 2 \mathrm{H}, J=15$ $\mathrm{Hz},-\mathrm{CH}=\mathrm{CH}-), 7.63\left(\mathrm{~d}, J=9 \mathrm{~Hz}, 4 \mathrm{H},-\mathrm{C}_{6}\right.$ $\mathrm{H}_{4}-$ ), $7.84\left(\mathrm{~d}, J=9 \mathrm{~Hz}, 4 \mathrm{H},-\mathrm{C}_{6} \mathrm{H}_{4}-\right), 9.97$ (s, $2 \mathrm{H},-\mathrm{CHO}$ ). Elem. Anal. Calcd. for $\mathrm{C}_{56} \mathrm{H}_{66} \mathrm{O}_{4}$ : C, $79.64 \%$; H, 7.10\%. Found: C, $79.60 \%$; H, $7.18 \%$.
1,4-Bis(4-formylstyryl)-2,5-bis(octyloxy)benzene (M3). Yield: $78 \% .{ }^{1} \mathrm{H} \mathrm{NMR}\left(300 \mathrm{MHz}, \mathrm{CDCl}_{3}, \delta\right)$ : $0.98\left(\mathrm{t}, \quad 6 \mathrm{H},-\mathrm{CH}_{3}\right), \quad 1.27-1.56[\mathrm{~m}, 20 \mathrm{H}$, $\left.-\left(\mathrm{CH}_{2}\right)_{n}-\right], 1.72-1.82\left(\mathrm{~m}, 4 \mathrm{H},-\mathrm{CH}_{2}-\right), 4.06(\mathrm{t}$, $\left.J=6.6 \mathrm{~Hz}, 4 \mathrm{H},-\mathrm{OCH}_{2}-\right), 7.12(\mathrm{~s}, 2 \mathrm{H}$, $\left.-\mathrm{C}_{6} \mathrm{H}_{2}-\right), 7.15(\mathrm{~d}, J=18 \mathrm{~Hz}, 2 \mathrm{H},-\mathrm{CH}=\mathrm{CH}-)$, $7.58(\mathrm{~d}, J=15 \mathrm{~Hz}, 2 \mathrm{H},-\mathrm{CH}=\mathrm{CH}-), 7.64(\mathrm{~d}, J$ $\left.=9 \mathrm{~Hz}, 4 \mathrm{H},-\mathrm{C}_{6} \mathrm{H}_{4}-\right), 7.84(\mathrm{~d}, J=9 \mathrm{~Hz}, 4 \mathrm{H}$, $-\mathrm{C}_{6} \mathrm{H}_{4}-$ ) 9.97 (s, 2H, - CHO). Elem. Anal. Calcd. for $\mathrm{C}_{56} \mathrm{H}_{66} \mathrm{O}_{4}$ : C, 80.77\%; H, 8.47\%. Found: C, $80.32 \%$; H, $8.36 \%$.

1,4-Bis(4-formylstyryl)-2,5-bis(dodecyloxy)benzene (M4). Yield: $62 \% .{ }^{1} \mathrm{H} \mathrm{NMR}\left(300 \mathrm{MHz}, \mathrm{CDCl}_{3}, \delta\right)$ : 0.89-0.94 (m, 12H, $\left.-\mathrm{CH}_{3}\right), 1.31-1.60[\mathrm{~m}, 12 \mathrm{H}$, $-\left(\mathrm{CH}_{2}\right)_{n}-\mathrm{]}, 1.61-1.70(\mathrm{~m}, 2 \mathrm{H},-\mathrm{CH}-), 4.08(\mathrm{t}$, $\left.J=6.6 \mathrm{~Hz}, 4 \mathrm{H},-\mathrm{OCH}_{2}-\right), 7.14(\mathrm{~s}, 2 \mathrm{H}$, $\left.-\mathrm{C}_{6} \mathrm{H}_{2}-\right), 7.17(\mathrm{~d}, J=18 \mathrm{~Hz}, 2 \mathrm{H},-\mathrm{CH}=\mathrm{CH}-)$, $7.60(\mathrm{~d}, J=15 \mathrm{~Hz}, 2 \mathrm{H},-\mathrm{CH}=\mathrm{CH}-), 7.68(\mathrm{~d}$, $\left.J=6 \mathrm{~Hz}, 4 \mathrm{H},-\mathrm{C}_{6} \mathrm{H}_{4}-\right), 7.86(\mathrm{~d}, J=6 \mathrm{~Hz}, 4 \mathrm{H}$, $-\mathrm{C}_{6} \mathrm{H}_{4}-$ ), 9.99 ( $\mathrm{s}, 2 \mathrm{H},-\mathrm{CHO}$ ). Elem. Anal. Calcd. for $\mathrm{C}_{72} \mathrm{H}_{98} \mathrm{O}_{4}$ : C, $81.54 \%$; $\mathrm{H}, 9.41 \%$. Found: C, $81.17 \%$; H, $9.00 \%$. 
1,4-Bis(4-formylstyryl)-2,5-bis(2-ethylhexyloxy) benzene (M5). Yield: $40 \% .{ }^{1} \mathrm{H}$ NMR $(300 \mathrm{MHz}$, $\left.\mathrm{CDCl}_{3}, \delta\right): 0.89-0.94\left(\mathrm{~m}, 12 \mathrm{H},-\mathrm{CH}_{3}\right), 1.31-1.60$ $\left[\mathrm{m}, 16 \mathrm{H},-\left(\mathrm{CH}_{2}\right)_{n}-\right], 1.61-1.70(\mathrm{~m}, 2 \mathrm{H},-\mathrm{CH}-)$, $3.97\left(\mathrm{t}, J=6.6 \mathrm{~Hz}, 4 \mathrm{H},-\mathrm{OCH}_{2}-\right), 7.14(\mathrm{~s}, 2 \mathrm{H}$, $\left.-\mathrm{C}_{6} \mathrm{H}_{2}-\right), 7.19(\mathrm{~d}, J=15 \mathrm{~Hz}, 2 \mathrm{H},-\mathrm{CH}=\mathrm{CH}-)$, $7.62(\mathrm{~d}, J=15 \mathrm{~Hz}, 2 \mathrm{H},-\mathrm{CH}=\mathrm{CH}-), 7.68(\mathrm{~d}$, $\left.J=6 \mathrm{~Hz}, 4 \mathrm{H},-\mathrm{C}_{6} \mathrm{H}_{4}-\right), 7.86(\mathrm{~d}, J=9 \mathrm{~Hz}, 4 \mathrm{H}$, $-\mathrm{C}_{6} \mathrm{H}_{4}-$ ), 10.00 (s, 2H, - CHO). Elem. Anal. Calcd. for $\mathrm{C}_{64} \mathrm{H}_{82} \mathrm{O}_{4}$ : C, $80.77 \% ; \mathrm{H}, 8.47 \%$. Found: C, $80.64 \%$; H, $8.31 \%$.

$\mathrm{PBVOC}_{8}-\mathrm{OC}_{4}$ (15). Yield: $74 \% .{ }^{1} \mathrm{H}$ NMR (300 $\left.\mathrm{MHz} \mathrm{CDCl}_{3}, \delta\right): 0.86\left(\mathrm{~m}, 12 \mathrm{H},-\mathrm{CH}_{3}\right), 1.23-1.82$ $\left[\mathrm{m}, 16 \mathrm{H},-\left(\mathrm{CH}_{2}\right)_{n}-\right], 3.95-4.04\left(\mathrm{~m}, 8 \mathrm{H},-\mathrm{OCH}_{2}-\right)$, 6.73-7.18 $\left(\mathrm{m}, 12 \mathrm{H},-\mathrm{C}_{6} \mathrm{H}_{4}-,-\mathrm{CH}=\mathrm{CH}-\right.$, overlapping), $7.26-7.60\left(\mathrm{~m}, 14 \mathrm{H},-\mathrm{C}_{6} \mathrm{H}_{4}-,-\mathrm{CH}\right.$ $=\mathrm{CH}-$, overlapping).

$\mathrm{PBVOC}_{8^{-}} \mathrm{OC}_{8}$ (16). Yield: 70\%. ${ }^{1} \mathrm{H}$ NMR (300 $\left.\mathrm{MHz}, \mathrm{CDCl}_{3}, \delta\right): 0.89\left(\mathrm{~m}, 12 \mathrm{H},-\mathrm{CH}_{3}\right), 1.23-1.86$ $\left[\mathrm{m}, 36 \mathrm{H},-\left(\mathrm{CH}_{2}\right)_{n}-\right], 3.98-4.05\left(\mathrm{~m}, 8 \mathrm{H},-\mathrm{OCH}_{2}-\right)$, 6.78-7.21 (m, $12 \mathrm{H},-\mathrm{C}_{6} \mathrm{H}_{4}-,-\mathrm{CH}=\mathrm{CH}-$, overlapping), $7.26-7.48\left(\mathrm{~m}, 14 \mathrm{H},-\mathrm{C}_{6} \mathrm{H}_{4}-,-\mathrm{CH}\right.$ $=\mathrm{CH}-$, overlapping).

$\mathrm{PBVOC}_{8^{-}} \mathrm{OC}_{12}$ (17). Yield: $50 \% .{ }^{1} \mathrm{H}$ NMR (300 $\left.\mathrm{MHz}, \mathrm{CDCl}_{3}, \delta\right): 0.84\left(\mathrm{~m}, 12 \mathrm{H},-\mathrm{CH}_{3}\right), 1.26-1.87$ $\left[\mathrm{m}, 48 \mathrm{H},-\left(\mathrm{CH}_{2}\right)_{n}-\right], 3.93-4.05\left(\mathrm{~m}, 8 \mathrm{H},-\mathrm{OCH}_{2}-\right)$, 6.78-7.21 $\left(\mathrm{m}, 12 \mathrm{H},-\mathrm{C}_{6} \mathrm{H}_{4}-,-\mathrm{CH}=\mathrm{CH}-\right.$, overlapping), $7.26-7.49\left(\mathrm{~m}, 14 \mathrm{H},-\mathrm{C}_{6} \mathrm{H}_{4}-,-\mathrm{CH}\right.$ $=\mathrm{CH}-$, overlapping).

$\mathrm{PBVOC}_{8^{-}} \mathrm{OEh}$ (18). Yield: $65 \% .{ }^{1} \mathrm{H}$ NMR (300 $\left.\mathrm{MHz}, \mathrm{CDCl}_{3}, \delta\right): 0.88\left(\mathrm{~m}, 18 \mathrm{H},-\mathrm{CH}_{3}\right), 1.18-1.80$ $\left[\mathrm{m}, 26 \mathrm{H},-\left(\mathrm{CH}_{2}\right)_{n}-\right], 3.95-3.97\left(\mathrm{~m}, 8 \mathrm{H},-\mathrm{OCH}_{2}-\right)$, $6.75-7.23\left(\mathrm{~m}, 12 \mathrm{H},-\mathrm{C}_{6} \mathrm{H}_{4}-,-\mathrm{CH}=\mathrm{CH}-\right.$, overlapping), $7.26-7.52\left(\mathrm{~m}, 14 \mathrm{H},-\mathrm{C}_{6} \mathrm{H}_{4}-,-\mathrm{CH}\right.$ $=\mathrm{CH}-$, overlapping).

\section{RESULTS AND DISCUSSION}

\section{Thermal Properties}

The thermal behavior and phase-transition temperatures of alkoxy-substituted OPVs (1-14) and poly( $p$-phenylene-vinylene)s (15-18), which were characterized with differential scanning calorimetry (DSC) and polarizing optical microscopy (POM) measurements, are listed in Tables 1-3. For three-conjugated phenyl oligomers (i.e., BIII series), the melting temperature $\left(T_{\mathrm{m}}\right)$ and crystallization temperature $\left(T_{\mathrm{cr}}\right)$ are demonstrated in
Table 1. Thermal Properties of the BIII Derivatives

\begin{tabular}{|c|c|}
\hline Compound & $\begin{array}{l}\text { Phase-Transition } \\
\text { Temperature }\left({ }^{\circ} \mathrm{C}\right)^{\mathrm{a}}\end{array}$ \\
\hline \multirow{2}{*}{ BIII-OMe (1) } & $177.7(68.2)$ \\
\hline & $156.3(-75.2)$ \\
\hline \multirow{2}{*}{ BIIIMe-OMe (2) } & $190.3(85.4)$ \\
\hline & $174.7(-86.5)$ \\
\hline \multirow{2}{*}{ BIIIOMe-OMe (3) } & $219.2(118.2)$ \\
\hline & $204.8(-118.6)$ \\
\hline \multirow{2}{*}{$\mathrm{BIIIOC}_{8}-\mathrm{OMe}(\mathbf{4})$} & $137.4(107.8)$ \\
\hline & $118.4(-112)$ \\
\hline \multirow{2}{*}{$\mathrm{BIIIOC}_{12}-\mathrm{OMe}(\mathbf{5})$} & $123.1(98.1)$ \\
\hline & $104.0(-90.6)$ \\
\hline \multirow{2}{*}{$\mathrm{BIIIOC}_{8}-\mathrm{OC}_{8}(\boldsymbol{6})$} & $86.2(41.4)$ \\
\hline & $73.0(-43.4)$ \\
\hline
\end{tabular}

a The corresponding enthalpy $(\mathrm{J} / \mathrm{g})$ is shown in parentheses. $\mathrm{K}=$ crystalline phase; $\mathrm{I}=$ isotropic liquid.

the following order: $\mathbf{3}>\mathbf{2}>\mathbf{1}>\mathbf{4}>\mathbf{5}>\mathbf{6}$ (shown in Table 1). In general, because of the weaker intermolecular interaction induced by the longer flexible chains, the longer alkoxy groups either on the central rings or on both end rings of the conjugated cores cause the reduction of the phase-transition temperatures (i.e., $T_{\mathrm{m}}$ and $T_{\mathrm{cr}}$ ) in the BIII series of three-conjugated ring oligomers (1-6). For instance, their phase-transition temperatures (i.e., $T_{\mathrm{m}}$ and $T_{\mathrm{cr}}$ ) are in the order of $\mathbf{3}>\mathbf{4}$ $>\mathbf{5}$ with the length of alkoxy terminal chains increasing (on both end rings) and with the length of lateral alkoxy chains $(-\mathrm{OMe})$ kept the same (on the central ring) or in the order of $4>6$ with the length of alkoxy side chains increasing (on the central ring) but with the length of alkoxy terminal chains $\left(-\mathrm{OC}_{8}\right)$ kept the same (on both end rings). As for $\mathbf{3}>\mathbf{2}>\mathbf{1}$ in $T_{\mathrm{m}}$ and $T_{\mathrm{cr}}$, it can be explained by the fact that the short terminal groups of - OMe and - Me groups in $\mathbf{3}$ and $\mathbf{2}$ generate larger molecular weights than that of $\mathbf{1}$ without terminal groups, and this accounts for the lowest transition temperature in $\mathbf{1}$. In addition, comparing $\mathbf{3}$ with $\mathbf{2}$, we find that methoxy groups containing oxygens have higher polarity and electronic density than methyl groups, which produce the highest $T_{\mathrm{m}}$ and $T_{\text {cr }}$ values in $\mathbf{3}$. 
Table 2. Thermal Properties of the BV Derivatives

\begin{tabular}{|c|c|c|c|}
\hline Compound & & ition Tempe & \\
\hline \multirow{2}{*}{$\mathrm{BVOC}_{4}-\mathrm{OEh}(\mathbf{7})$} & $185.6(40.1)$ & $253.9(0.9)$ & \\
\hline & $168.5(-40.1)$ & $242.0(-1.9)$ & \\
\hline \multirow{2}{*}{$\mathrm{BVOC}_{4}-\mathrm{OC}_{8}(\mathbf{8})$} & $184.2(43.0)$ & $274.7(2.2)$ & \\
\hline & $156.8(-41.2)$ & $266.6(-1.9)$ & \\
\hline \multirow{2}{*}{$\mathrm{BVOC}_{4}-\mathrm{OC}_{12}(\mathbf{9})$} & $79.1(11.6)$ & $185.6(43.2)$ & $222.5(1.7)$ \\
\hline & $74.7(-11.9)$ & $164.8(-43.2)$ & $220.0(-1.4)$ \\
\hline \multirow{2}{*}{$\mathrm{BVOC}_{8}-\mathrm{OEh}(\mathbf{1 0})$} & $165.3(64.9)$ & $255.2(2.3)$ & \\
\hline & $139.2(-43.0)$ & $219.8(-5.6)$ & \\
\hline \multirow{2}{*}{$\mathrm{BVOC}_{8}-\mathrm{OC}_{8}(\mathbf{1 1})$} & $145.8(49.6)$ & $250.9(2.3)$ & \\
\hline & $\overline{121.5(-44.9)}$ & $248.5(-2.1)$ & \\
\hline \multirow{2}{*}{$\mathrm{BVOC}_{8}-\mathrm{OC}_{12}(\mathbf{1 2})$} & $155.2(51.6)$ & $203.7(1.1)$ & \\
\hline & $132.6(-52.7)$ & $201.0(-1.3)$ & \\
\hline \multirow{2}{*}{$\mathrm{BVOC}_{12}-\mathrm{OC}_{8}(\mathbf{1 3})$} & $117.8(2.9)$ & $141.3(36.2)$ & $189.5(0.4)$ \\
\hline & $\mathbf{n}$ & & $187.0(-0.5)$ \\
\hline \multirow{2}{*}{$\mathrm{BVOC}_{12}-\mathrm{OC}_{12}(\mathbf{1 4})$} & $157.3(49.4)$ & $188.3(1.0)$ & \\
\hline & $\overline{142.6(-51.4)}$ & $\overline{186.6(-1.1)}$ & \\
\hline \multirow{2}{*}{$\mathrm{BV}^{-\mathrm{OC}_{8}}{ }^{\mathrm{b}}$} & $183.0(77.0)$ & $204.0(1.0)$ & \\
\hline & 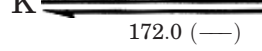 & $202.0(-)$ & \\
\hline
\end{tabular}

a The corresponding enthalpy $(\mathrm{J} / \mathrm{g})$ is shown in parentheses. $\mathrm{K}=$ crystalline phase; $\mathrm{N}=$ nematic phase; $\mathrm{I}=$ isotropic liquid.

${ }^{\mathrm{b}}$ As a comparison from ref. 53 .

Similar effects on $T_{\mathrm{m}}$ and the isotropization temperature $\left(T_{\mathrm{i}}\right)$ can also be observed in the fiveconjugated ring series (i.e., BV series); besides that, mesomorphism is introduced into the BV series (shown in Table 2) by the replacement of three-conjugated rings with five-conjugated phenyl cores. Compared with $\mathbf{8}, \mathbf{1 1}$, and 13, compound $\mathrm{BV}-\mathrm{OC}_{8}$ without terminal groups on both end rings of the cores, which was synthesized and reported by Hadziioannou et al., ${ }^{53}$ possesses the narrowest mesophasic range $\left(21^{\circ} \mathrm{C}\right.$ on heating $)$ in the BV series with the same five-conjugated phenyl cores and lateral $-\mathrm{OC}_{8}$ alkoxy substituents in Table 2. Hence, the introduction of alkoxy terminal chains on both end rings of the conjugated cores may enhance the stability of the mesophase (the nematic phase). However, according to a comparison of $\mathbf{1 3}$ with 11, elongating the alkoxy terminal chains on both end rings of the cores from $-\mathrm{OC}_{8}$ to $-\mathrm{OC}_{12}$ would weaken the longitu- dinal force, which makes the nematic phase become more unfavorable in 13. Similar results, that is, the most proper alkoxy terminal chains (on both end rings) of $\mathrm{BVOC}_{8}$, also occur in analogous $\mathrm{BVOC}_{n}-\mathrm{OC}_{12}$ and $\mathrm{BVOC}_{n}$-OEh systems containing lateral $-\mathrm{OC}_{12}$ and $-\mathrm{OEh}$ groups as side chains (on the central ring). Once the most suitable terminal chains ( $-\mathrm{OC}_{8}$; on both end rings) of $\mathrm{BVOC}_{8}$ are chosen, 10, 11, and $\mathbf{1 2}$ analogues can be compared to justify the most favorable side-chain length on the central ring. Again, elongating or branching the alkoxy side chains (on the central ring) from $-\mathrm{OC}_{8}$ to $-\mathrm{OC}_{12}$ or from $-\mathrm{OC}_{8}$ to $-\mathrm{OEh}$ would weaken the favorable force to form the nematic phase. Therefore, 11 has the most appropriate chain length on both ends (on both end rings) and sides (on the central ring) of the conjugated core and thus has the widest mesophasic range $\left(105{ }^{\circ} \mathrm{C}\right.$ on heating) in the BV series (7-14) in Table 2. 
Table 3. Thermal Properties of the PBV Polymers

\begin{tabular}{|c|c|c|c|}
\hline Compound & $\begin{array}{l}\text { Phase-Transi } \\
\text { Temperature }\end{array}$ & tion & $T_{\mathrm{d}}\left({ }^{\circ} \mathrm{C}\right)^{\mathrm{b}}$ \\
\hline $\mathrm{PBVOC}_{8}-\mathrm{OC}_{4}(\mathbf{1 5})$ & $\mathrm{G} \stackrel{75}{\rightleftharpoons} \mathrm{K} \underset{112}{\stackrel{130}{\rightleftharpoons}} \mathrm{N}$ & $\underset{260}{\stackrel{280}{\rightleftharpoons}} \mathrm{I}$ & 411 \\
\hline $\mathrm{PBVOC}_{8}-\mathrm{OC}_{8}(\mathbf{1 6})$ & $\mathrm{G} \stackrel{54}{\rightleftharpoons} \mathrm{K} \underset{104}{\stackrel{117}{\rightleftharpoons}} \mathrm{N}$ & $\underset{220}{\stackrel{230}{\rightleftharpoons}} \mathrm{I}$ & 409 \\
\hline $\mathrm{PBVOC}_{8}-\mathrm{OC}_{12}(\mathbf{1 7})$ & $\mathrm{G} \stackrel{85}{\rightleftharpoons} \mathrm{K} \underset{100}{\stackrel{110}{\rightleftharpoons}} \mathrm{N}$ & $\underset{235}{\stackrel{245}{\rightleftharpoons}} \mathrm{I}$ & 393 \\
\hline $\mathrm{PBVOC}_{8}-\mathrm{OEh}(\mathbf{1 8})$ & $\mathrm{G} \stackrel{85}{\rightleftharpoons} \mathrm{K} \underset{115}{\stackrel{125}{\rightleftharpoons}} \mathrm{N}$ & $\underset{245}{\stackrel{255}{\rightleftharpoons}} \mathrm{I}$ & 400 \\
\hline
\end{tabular}

${ }^{a}$ The phase-transition temperatures were obtained from POM. $\mathrm{G}=$ glassy state; $\mathrm{K}=$ crystalline phase; $\mathrm{N}=$ nematic phase; $\mathrm{I}=$ isotropic liquid.

${ }^{b}$ Temperature of $5 \%$ weight loss measured by TGA in nitrogen (i.e., the thermal decomposition temperature).

On the basis of the same consideration of the widest mesophasic ranges in $\mathrm{BVOC}_{8}$ derivatives, analogous polymers, that is, the PBV series (1518), containing various side groups on the central rings, were synthesized in this study. Their thermal properties are shown in Table 3; and their molecular weights and PDIs are listed in Table 4. Similar phase behavior, that is, a glass-transition temperature $\left(T_{\mathrm{g}}\right)$ and a nematic phase, of these PBV derivatives, can be observed (see Table 3). Because of the variation of the molecular weights and broadened transition temperatures (determined by POM) of the polymers, the alkoxy sidegroup effect on the polymers is not so obvious as that on their oligo-analogues. The temperature ranges of the LC phase (i.e., the nematic phase) of the polymers are wider than those of analogous oligomers because the rigid cores of the polymer backbones are connected end to end by the flexible alkoxy spacers, which enhance the mesogenic longitudinal interaction in the nematic phase. Accordingly, because of the introduction of flexible alkoxy groups either on the central rings or on both end rings of the conjugated cores, not only the solubility but also the thermal properties (including the phase behavior and phase-transition temperatures, i.e., $T_{\mathrm{m}}$ and $T_{\mathrm{i}}$ ) of both threeconjugated and five-conjugated ring derivatives can be adjusted.

\section{Optical Properties}

The ultraviolet-visible (UV-vis) absorption spectra of these compounds in solution states (di- chloroethane as the solvent) were measured, and their maximum emission wavelength $\left(\lambda_{\max }\right)$ values are listed in Table 5. The peaks of UV-vis spectra are near 393-398 nm for three-conjugated ring oligomers (1-5), 423-431 nm for fiveconjugated ring oligomers (7-14), and near 422$423 \mathrm{~nm}$ for five-conjugated ring polymers (1518). In comparison with three-conjugated ring oligomers, five-conjugated ring derivatives have larger $\lambda_{\max }$ values, that is, redshifted absorption, because of the smaller energy gaps of longer conjugations in five-conjugated ring derivatives. Similar absorption $\lambda_{\max }$ values have been observed for both oligomers and polymers of fiveconjugated ring derivatives because of their similar conjugation lengths in solutions.

The PL and EL spectra of these compounds in solutions (with dichloroethane as the solvent) and films were measured, and their $\lambda_{\max }$ values are also listed in Table 5; the PL measurements were excited at an emission wavelength of 285 $\mathrm{nm}$. The $\lambda_{\max }$ values of PL and EL spectra of analogous derivatives $\mathbf{6 , 1 1}$, and $\mathbf{1 6}$ (shown in Table 5) are as follows: for three-conjugated ring oligomer 6. $\lambda_{\max }(\mathrm{PL})$ is 447,503 , and $448 \mathrm{~nm}$ in solutions, pure films, and blend films, respectively, and $\lambda_{\text {max }}(\mathrm{EL})$ is $459 \mathrm{~nm}$ in blend films; for five-conjugated ring oligomer $11, \lambda_{\max }(\mathrm{PL})$ is 487,532 , and $498 \mathrm{~nm}$ in solutions, pure films, and blend films, respectively, and $\lambda_{\max }(\mathrm{EL})$ is $504 \mathrm{~nm}$ in blend films; and for five-conjugated ring polymer 16, $\lambda_{\max }(\mathrm{PL})$ is 486,545 , and $496 \mathrm{~nm}$ in solutions, pure films, and blend films, respectively, and $\lambda_{\max }(\mathrm{EL})$ is $497 \mathrm{~nm}$ in blend films. All the aforementioned blend films are solid solutions with PVK as the solvent in a dopant/PVK ratio of $1: 100 \mathrm{wt} \%$. Because the role of the PVK matrix in the blend films is similar to that of the solvent in the solution, the blend films are solid solutions that show the same effect as the solutions with high concentrations of chromophores. A comparison of the $\lambda_{\max }(\mathrm{PL})$ values in solutions, pure films, and blend films of analogous derivatives $\mathbf{6}, \mathbf{1 1}$,

Table 4. Molecular Weights and PDIs of the PBV Polymers

\begin{tabular}{crc}
\hline \multicolumn{1}{c}{ Compound } & \multicolumn{1}{c}{$M_{\mathrm{w}}$} & PDI $\left(M_{\mathrm{w}} / M_{\mathrm{n}}\right)$ \\
\hline PBVOC $_{8}-\mathrm{OC}_{4}(\mathbf{1 5})$ & 11,778 & 1.7 \\
$\mathrm{PBVOC}_{8}-\mathrm{OC}_{8}(\mathbf{1 6})$ & 7,240 & 1.3 \\
$\mathrm{PBVOC}_{8}-\mathrm{OC}_{12}(\mathbf{1 7})$ & 10,193 & 1.4 \\
$\mathrm{PBVOC}_{8}-\mathrm{OEh}(\mathbf{1 8})$ & 15,166 & 1.8 \\
\hline
\end{tabular}


Table 5. UV-vis, PL, ${ }^{a}$ and EL Properties of BIII, BV, and PBV Derivatives

\begin{tabular}{|c|c|c|c|c|c|}
\hline Compound & $\begin{array}{c}\text { UV-vis } \\
(\text { Absorption })^{\mathrm{a}}\end{array}$ & $\begin{array}{c}\text { PL } \\
(\text { Solution })^{b}\end{array}$ & $\begin{array}{c}\lambda_{\max }(\mathrm{nm}) \\
\text { for PL } \\
\text { (Pure Film) }\end{array}$ & $\begin{array}{c}\text { PL } \\
\text { (Blend } \\
\text { Film) }{ }^{c}\end{array}$ & $\begin{array}{c}\text { EL } \\
\text { (Blend } \\
\text { Film) }^{\mathrm{c}}\end{array}$ \\
\hline BIII-OMe (1) & 393 & 442 & 474 & 444 & 449 \\
\hline BIIIMe-OMe (2) & 394 & $441(461)^{\mathrm{e}}$ & 477 & 444 & 449 \\
\hline BIIIOMe-OMe (3) & 397 & $444(465)$ & 495 & 448 & 447 \\
\hline $\mathrm{BIIIOC}_{8}-\mathrm{OMe}(\mathbf{4})$ & 398 & $445(467)$ & $501(527)$ & 453 & 450 \\
\hline $\mathrm{BIIIOC}_{12}-\mathrm{OMe}(\mathbf{5})$ & 397 & $445(466)$ & $501(529)$ & 447 & 456 \\
\hline $\mathrm{BIIIOC}_{8}-\mathrm{OC}_{8}(\mathbf{6})$ & 398 & $447(470)$ & $503(538)$ & 448 & 459 \\
\hline $\mathrm{BVOC}_{4}$-OEh (7) & 426 & $483(513)$ & 530 & 508 & 504 \\
\hline $\mathrm{BVOC}_{4}-\mathrm{OC}_{8}(\mathbf{8})$ & 424 & $483(512)$ & 528 & 503 & 501 \\
\hline $\mathrm{BVOC}_{4}-\mathrm{OC}_{12}(\mathbf{9})$ & 423 & $486(514)$ & 528 & 510 & 503 \\
\hline $\mathrm{BVOC}_{8}-\mathrm{OEh}(\mathbf{1 0})$ & 431 & $486(513)$ & 526 & 525 & 510 \\
\hline $\mathrm{BVOC}_{8}-\mathrm{OC}_{8}(\mathbf{1 1})$ & 431 & $487(512)$ & $532(567)$ & 498 & 504 \\
\hline $\mathrm{BVOC}_{8}-\mathrm{OC}_{12}(\mathbf{1 2})$ & 431 & $487(514)$ & 537 & 492 & 504 \\
\hline $\mathrm{BVOC}_{12}-\mathrm{OC}_{8}(\mathbf{1 3})$ & 430 & $487(512)$ & 529 & 497 & 493 \\
\hline $\mathrm{BVOC}_{12}-\mathrm{OC}_{12}(\mathbf{1 4})$ & 431 & $487(514)$ & 542 & 486 & 489 \\
\hline $\mathrm{PBVOC}_{8}-\mathrm{OC}_{4}(\mathbf{1 5})$ & 423 & $491(510)$ & 543 & 492 & 495 \\
\hline $\mathrm{PBVOC}_{8}-\mathrm{OC}_{8}(\mathbf{1 6})$ & 422 & $486(511)$ & 545 & 496 & 497 \\
\hline $\mathrm{PBVOC}_{8}-\mathrm{OC}_{12}(\mathbf{1 7})$ & 422 & $493(512)$ & 544 & 492 & 499 \\
\hline $\mathrm{PBVOC}_{8}-\mathrm{OEh}(\mathbf{1 8})$ & 423 & $490(511)$ & 531 & 495 & 498 \\
\hline $\mathrm{BV}-\mathrm{OC}_{8}^{\mathrm{d}}$ & 422 & 483 & 529 & - & - \\
\hline
\end{tabular}

and $\mathbf{1 6}$ shows that their $\lambda_{\max }(\mathrm{PL})$ values are all in the order of pure films $>$ blend films $>$ solutions. This can be explained by the fact that higher degrees of aggregation take place in solid solutions of pure and blend films, which contain higher concentrations of chromophores. Compared with pure films, solid solutions of blend films lead to reduced intermolecular interactions, and fewer aggregate states of blend films occur, so fewer redshifted emissions are produce. The redshifts of $\lambda_{\max }(\mathrm{PL})$ values in pure and blend films of analogous derivatives $\mathbf{6}, \mathbf{1 1}$, and $\mathbf{1 6}$ are comparable, so their extents of aggregation in pure and blend films are similar. Besides, their $\lambda_{\text {max }}(\mathrm{EL})$ values correspond to their $\lambda_{\text {max }}(\mathrm{PL})$ values in blend films because the aggregation levels in blend films are roughly equivalent in both EL and PL measurements. Therefore, the $\pi-\pi$ stacking effect appears in solid-state films to form excimers, so the redshifted phenomena were observed. The $\lambda_{\max }$ values of PL and EL spectra in pure PVK films are usually found around $400 \mathrm{~nm}$, and this emission wavelength is not so obvious in the PL and EL spectra of PVK-doped systems. Therefore, the PVK matrix might act as an energy-transfer component in the blend film systems.

As for three-conjugated ring oligomers in pure films, we cannot observe a second peak in the PL spectra of 1 (see Table 5). The second PL (pure film) peaks of $\mathbf{2}$ and $\mathbf{3}$ are also not obvious, but those of 4, 5, and 6 are easier to detect. Besides, the latter compounds with longer alkoxy terminal chains (on both end rings), that is, $\mathbf{4}, \mathbf{5}$, and $\mathbf{6}$, also have larger redshifts of $\lambda_{\max }$ values in PL (pure film) emissions in comparison with their corresponding PL (solution) emissions. This is possibly due to the fact that the longer and polar end alkoxy chains stack on each other to cause redshifts on the major PL peaks (i.e., $\lambda_{\max }$ ). The fact that stronger second peaks are caused in these compounds possibly originates from the excimers, as confirmed by the concentration study of the solution samples, that is, the gradual appearance of second peaks at higher concentrations. Additionally, compared with 1 and 2, 3, possessing the same core and lateral OMe groups, has a stronger redshifted PL emission in pure films, although all of them have similar $\lambda_{\max }$ values in PL (solution) emissions. This can be 


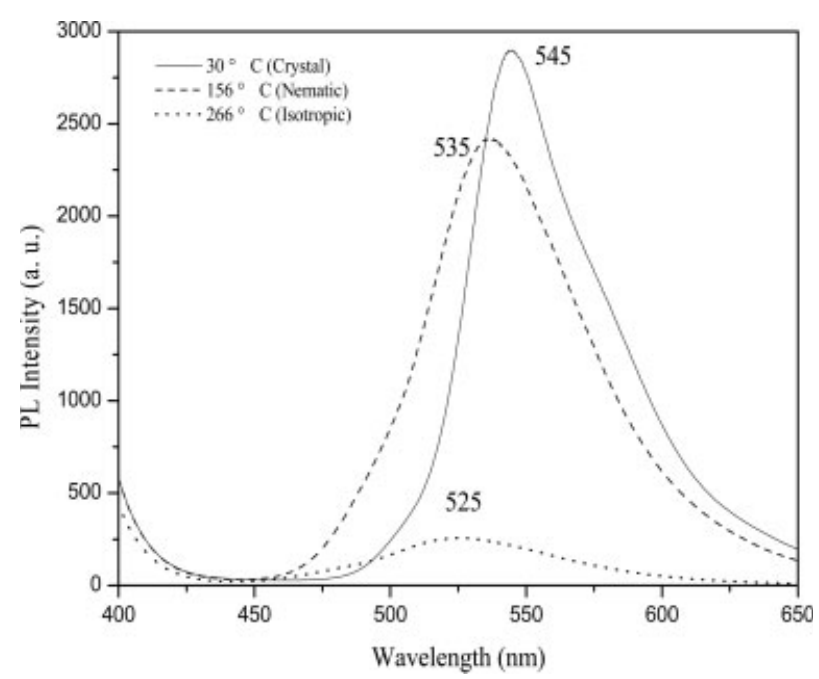

Figure 1. PL spectra of $\mathbf{1 1}$ in various phases: the crystalline state $\left(\lambda_{\max }=545 \mathrm{~nm}\right)$, LC state $\left(\lambda_{\max }\right.$ $=535 \mathrm{~nm})$, and isotropic state $\left(\lambda_{\max }=525 \mathrm{~nm}\right)$. The polarizer is parallel to the rubbing direction.

explained by the extra polar interactions of alkoxy chains on both ends of 3, which cause more aggregation. Similar aggregation and redshifts of $\lambda_{\max }$ values in PL emissions occur in pure films in comparison with the corresponding solutions of the BV and PBV series. Consequently, blend films of three-conjugated ring oligomers show blue PL and EL emissions ( $\lambda_{\max }=444-459$ $\mathrm{nm}$ ), and those of five-conjugated ring derivatives (oligomers and polymers) show blue-greenish PL and EL emissions $\left(\lambda_{\max }=486-525 \mathrm{~nm}\right.$ ).

Interestingly, the PL emissions of five-conjugated ring oligomers show different colors in different phases. Figure 1 shows the PL spectra of 11 via heating from the crystalline state $\left[\lambda_{\max }\right.$ $(\mathrm{PL})=545 \mathrm{~nm}]$ to the LC state $\left[\lambda_{\max }(\mathrm{PL})=535\right.$ $\mathrm{nm}]$ and sequentially to the isotropic phase $\left[\lambda_{\max }(\mathrm{PL})=525 \mathrm{~nm}\right]$. Because of larger aggregation caused by parallel alignment and larger film thickness inside the rubbing cell (with a $9-\mu \mathrm{m}$ cell gap) of Figure $1, \lambda_{\max }(\mathrm{PL})=545 \mathrm{~nm}$ of $\mathbf{1 1}$ in the aligned crystalline state (Fig. 1) is larger than $\lambda_{\max }(\mathrm{PL})=532 \mathrm{~nm}$ in the film state (ca. $40 \mathrm{~nm}$ via spin coating on a glass substrate) of $\mathbf{1 1}$ in Table 5. When $\mathbf{1 1}$ is heated serially from the crystalline phase to the isotropic phase, blueshifts in the PL spectra can be observed in Figure 1. One of the possible reasons for the blueshift of $\lambda_{\max }(\mathrm{PL})$ upon heating is that increasing the temperature may eliminate the coplanar configuration of the molecular structure and reduce its conjugation length (thus increasing the energy gap), so it shows blueshifts due to noncoplanar structures at higher temperatures. It is also possible that the aggregation level of molecular stacking decreases, and this causes the blueshift as the temperature increases. However, lower aggregation levels should have higher PL intensities, and this does not agree with the lowest PL emission possessed by the isotropic state in Figure 1. Hence, the order of the PL emission intensity (revealed in Fig. 1), that is, crystalline state $>$ LC state $>$ isotropic state, rules out the possibility of the blueshift of the isotropic state (with much lower PL intensity) caused by the lower aggregation level. In addition, the order of the full width at half-maximum (fwhm) values of various phases is as follows: fwhm (crystalline state) $=56$ $\mathrm{nm}<\mathrm{fwhm}($ LC state $)=70 \mathrm{~nm}<$ fwhm (isotropic state $)=76 \mathrm{~nm}$. It is conceived that broadened electron transition state distributions in various states of molecular architectures will have larger fwhm values, and this indicates that molecules possess more types of twisted conformations at higher temperatures. Because the blueshift of the isotropic state having the lowest PL intensity cannot be explained by the least aggregation of the isotropic phase, therefore, the least coplanar configuration with a wide distribution of conformations (as well as conjugation) in the isotropic phase may be more favorable for explaining the blueshift and the reduction of the PL intensity with the broadest fwhm value in the isotropic state.

As we know, if a sample possesses an LC phase, the LC phase can be aligned in a rubbing cell. When the rubbing cell is parallel to the polarizer, the conjugated core, which is aligned in the rubbing direction, will also be parallel to the polarizer at the same time. Therefore, a maximum PL emission can be observed when the polarizer is in the parallel direction of rubbing, and a minimum PL emission can be observed when the polarizer is in the perpendicular direction of rubbing. The polarized PL of $\mathbf{1 1}$ in the LC phase is shown in Figure 2. The polarization ratio is defined as $\mathrm{PL} / \mathrm{PL}_{\perp}$, where $\mathrm{PL} /$ and $\mathrm{PL}_{\perp}$ are the maximum PL emission intensities as the polarizer is parallel and perpendicular to the rubbing direction, respectively. Figure 3 shows the polarization ratio and $\lambda_{\max }(\mathrm{PL})$ values of $\mathbf{1 1}$ in rubbing cells at various temperatures. The polarization ratio reaches a maximum value at the transition of the crystalline phase to the mesophase upon heating, and the polarization ratio decreases as the temperature increases; this is similar to the 


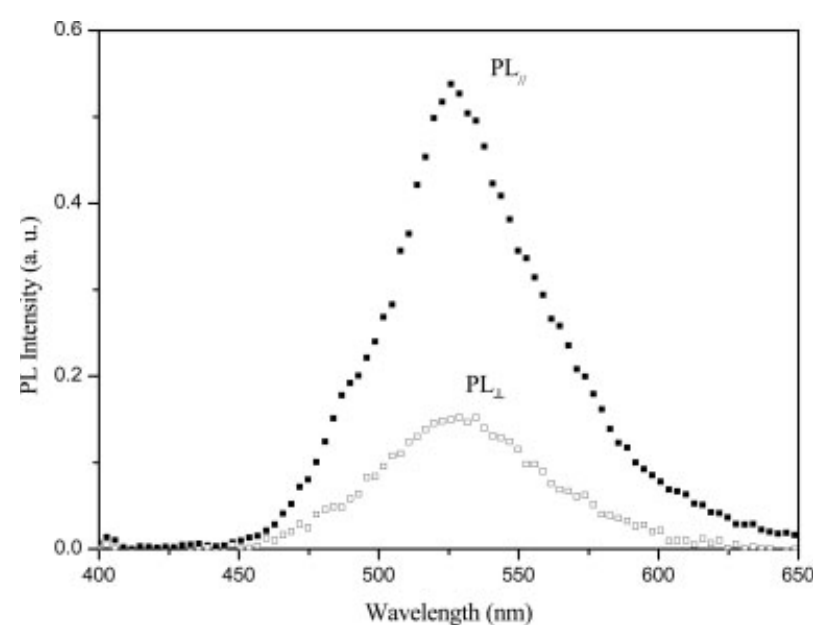

Figure 2. PL spectra of $\mathbf{1 1}$ at $156{ }^{\circ} \mathrm{C}$ (in the nematic phase).

behavior of the order parameter of the LC materials upon heating. As mentioned previously, blueshifts in PL spectra can be observed upon heating, and this is due to the reduction of coplanar configurations and conjugation lengths and thus to larger energy gaps at higher temperatures. Interestingly, the largest reduction of the $\lambda_{\max }$ value and the largest increase in the polarization ratio both occur at the transition temperatures around heating into the mesophase, and this illustrates that the alignment of the mesogenic cores by the surface may cause twisted configurations and noncoplanar conformations. The maximum polarization ratio and related optical properties [including HOMO/lowest unoccupied molecular orbital (LUMO) energy levels, optical band gaps, and PL quantum yield] of all the compounds are shown in Table 6 . The maximum polarization ratio of $\mathbf{1 1}$ is 3.6 (also shown in Fig. 2 ), and the highest value of the polarization ratio is 6.2 (found in 13). These values of oligomers are generally higher (except for 14, for which the polarization ratio is 2.5 ; this may be due to the symmetrical, long, flexible chains on both the sides and ends of the conjugated cores) than those of polymers (polarization ratio $=1.6-3.1$ for the PBV series) because polymer chains cannot be aligned completely in the rubbing direction (because of the higher viscosity and entanglement of polymer chains), and this induces the reduction of the polarization ratios in the polymers. Overall, the maximum polarization ratios of PL emissions are not correlated to the lengths of the side groups and end groups attached to the cores.
The LUMO values, which are calculated from the HOMO values (by AC-2) minus the optical band gaps (by UV-vis), are also listed in Table 6 . The band gaps of the three-conjugated ring oligomers (BIII series) are between 2.67 and $2.75 \mathrm{eV}$. Compared with three-conjugated ring oligomers, five-conjugated ring derivatives (BV oligomers and PBV polymers) possess smaller band gaps, between 2.51 and $2.57 \mathrm{eV}$, because of the longer conjugated length of five-conjugated ring derivatives, and thus generate longer wavelengths of PL and EL emissions. However, the band gaps of five-conjugated ring oligomers and analogous polymers are too indistinct to have divergent PL and EL emissions. The PL quantum yields (in solutions) of BIII oligomers, BV oligomers, and PBV polymers (shown in Table 6) are $0.48-0.56,0.84-0.95$, and $0.65-0.99$, respectively. Oligomers and polymers of five-conjugated ring derivatives have similar PL quantum yields. Accordingly, five-conjugated ring derivatives (BV oligomers and PBV polymers) have larger PL quantum yields than three-conjugated ring BIII oligomers, and this may be due to the longer conjugation lengths in five-conjugated ring systems. The PL and EL spectra of analogous derivatives $\mathbf{6 , 1 1}$, and 16 containing alkoxy groups $\left(-\mathrm{OC}_{8}\right)$ of the same length on both the sides and ends are compared in Figures 4-6, respectively. For these analogous derivatives, they induce variations of the energy gaps and luminescence behavior. In their solution and pure film states, two peaks have been found in the PL spectra (shown in Figs. 4 and 5) of oligomers 6 and 11, and a single peak has been found in the PL spectra (shown in Fig. 6) of polymer 16. Thus, more types of twisted

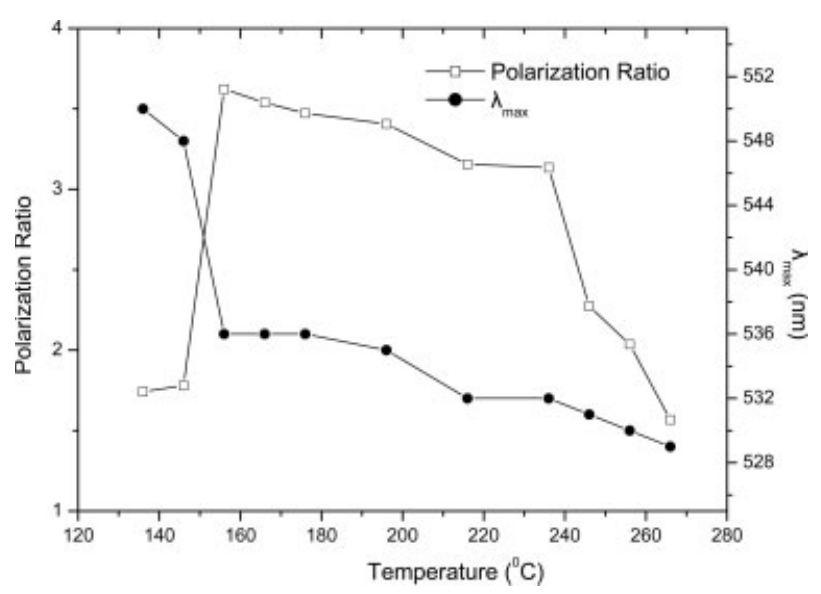

Figure 3. Polarization ratio and $\lambda_{\max }(\mathrm{PL})$ values of $\mathbf{1 1}$ in rubbing cells at various temperatures. 
Table 6. Optical Properties of the BIII, BV, and PBV Derivatives

\begin{tabular}{|c|c|c|c|c|c|}
\hline Compound & $\begin{array}{c}\text { HOMO/LUMO } \\
(\mathrm{eV})\end{array}$ & $\begin{array}{c}\text { Band } \\
\operatorname{Gap}(e V)^{a}\end{array}$ & $\begin{array}{c}\Phi(\mathrm{PL})^{\mathrm{b}} \\
(\mathrm{soln})\end{array}$ & $\begin{array}{c}\Phi(\mathrm{PL})^{\mathrm{c}} \\
(\text { film })\end{array}$ & $\begin{array}{c}\text { Maximum } \\
\text { Polarization } \\
\text { Ratio }^{\mathrm{d}}\end{array}$ \\
\hline BIII-OMe (1) & $5.63 / 2.92$ & 2.71 & 0.30 & 0.34 & NA \\
\hline BIIIMe-OMe (2) & $5.62 / 2.93$ & 2.69 & 0.30 & 0.34 & NA \\
\hline BIIIOMe-OMe (3) & $5.76 / 3.01$ & 2.75 & 0.27 & 0.33 & NA \\
\hline $\mathrm{BIIIOC}_{8}-\mathrm{OMe}(\mathbf{4})$ & $5.87 / 3.15$ & 2.72 & 0.33 & 0.36 & NA \\
\hline $\mathrm{BIIIOC}_{12}-\mathrm{OMe}(\mathbf{5})$ & $5.70 / 2.95$ & 2.75 & 0.26 & 0.31 & NA \\
\hline $\mathrm{BIIIOC}_{8}-\mathrm{OC}_{8}(\mathbf{6})$ & $5.61 / 2.86$ & 2.74 & 0.30 & 0.33 & NA \\
\hline $\mathrm{BVOC}_{4}-\mathrm{OEh}(\mathbf{7})$ & $5.62 / 3.06$ & 2.56 & 0.51 & 0.52 & 4.4 \\
\hline $\mathrm{BVOC}_{4}-\mathrm{OC}_{8}(\mathbf{8})$ & $5.45 / 2.89$ & 2.56 & 0.50 & 0.52 & 3.9 \\
\hline $\mathrm{BVOC}_{4}-\mathrm{OC}_{12}(\mathbf{9})$ & $5.61 / 3.05$ & 2.56 & 0.48 & 0.50 & 5.0 \\
\hline $\mathrm{BVOC}_{8}-\mathrm{OEh}(\mathbf{1 0})$ & $5.69 / 3.14$ & 2.55 & 0.45 & 0.49 & 4.5 \\
\hline $\mathrm{BVOC}_{8}-\mathrm{OC}_{8}(\mathbf{1 1})$ & $5.58 / 3.07$ & 2.51 & 0.52 & 0.52 & 3.6 \\
\hline $\mathrm{BVOC}_{8}-\mathrm{OC}_{12}(\mathbf{1 2})$ & $5.74 / 3.23$ & 2.51 & 0.49 & 0.52 & 4.8 \\
\hline $\mathrm{BVOC}_{12}-\mathrm{OC}_{8}(\mathbf{1 3})$ & $5.64 / 3.13$ & 2.51 & 0.51 & 0.52 & 6.2 \\
\hline $\mathrm{BVOC}_{12}-\mathrm{OC}_{12}(\mathbf{1 4})$ & $5.69 / 3.18$ & 2.51 & 0.47 & 0.50 & 2.5 \\
\hline $\mathrm{PBVOC}_{8}-\mathrm{OC}_{4}(\mathbf{1 5})$ & $5.98 / 3.42$ & 2.56 & 0.40 & 0.46 & 1.6 \\
\hline $\mathrm{PBVOC}_{8}-\mathrm{OC}_{8}(\mathbf{1 6})$ & $5.60 / 3.05$ & 2.55 & 0.35 & 0.44 & 1.7 \\
\hline $\mathrm{PBVOC}_{8}-\mathrm{OC}_{12}(\mathbf{1 7})$ & $5.59 / 3.03$ & 2.56 & 0.43 & 0.46 & 2.1 \\
\hline $\mathrm{PBVOC}_{8}-\mathrm{OEh}(\mathbf{1 8})$ & $5.68 / 3.11$ & 2.57 & 0.53 & 0.48 & 3.1 \\
\hline
\end{tabular}

a Optical band gap obtained by UV-vis.

${ }^{\mathrm{b}} \mathrm{PL}$ quantum yield in solutions with a concentration of $10^{-5} \mathrm{M}$ in dichloroethane and quinine sulfate as the reference with a quantum yield of 0.54.

${ }^{\mathrm{c}} \mathrm{PL}$ quantum yield in films with a weight ratio of 1:100 (with PVK as the matrix) and MEHPPV as the reference with a quantum yield of 0.22 .

${ }^{\mathrm{d}} \mathrm{NA}=$ not available because there is no LC phase.

conformations and conjugations or more aggregation, including vibronic motion, occurs in oligomers than in polymers. As shown in Table 3, the glassy state of reduced crystallinity in the PBV polymers has been observed in their thermal

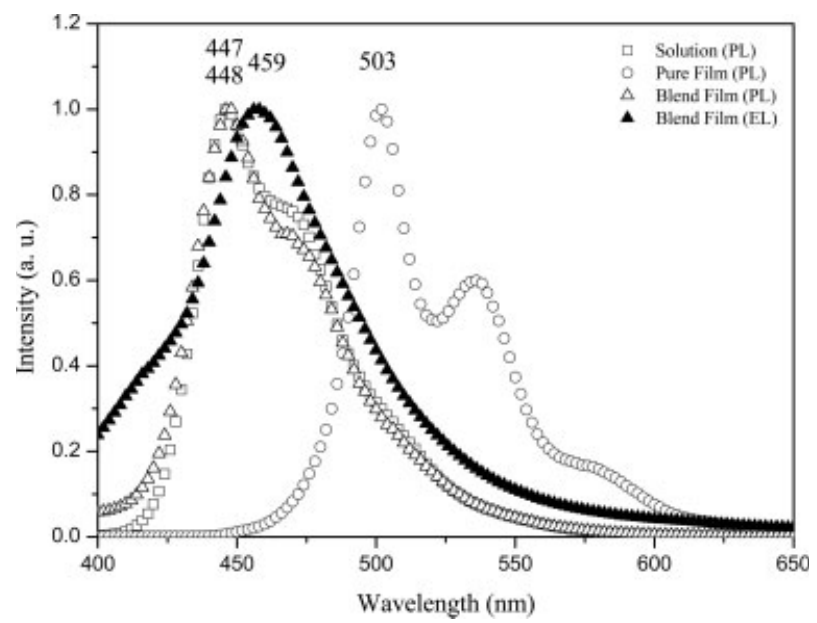

Figure 4. PL and EL spectra of 6 (the PVK matrix was used in the blend films; chromophore/PVK $=1: 100 \mathrm{wt} \%)$. properties (all possess $T_{\mathrm{g}}$ between 54 and $85{ }^{\circ} \mathrm{C}$ ) and has also been confirmed by XRD measurements (as glassy states with broadened peaks at ca. $21^{\circ}$ ), so the polymers have less crystallinity (i.e., less aggregation) than the oligomers.

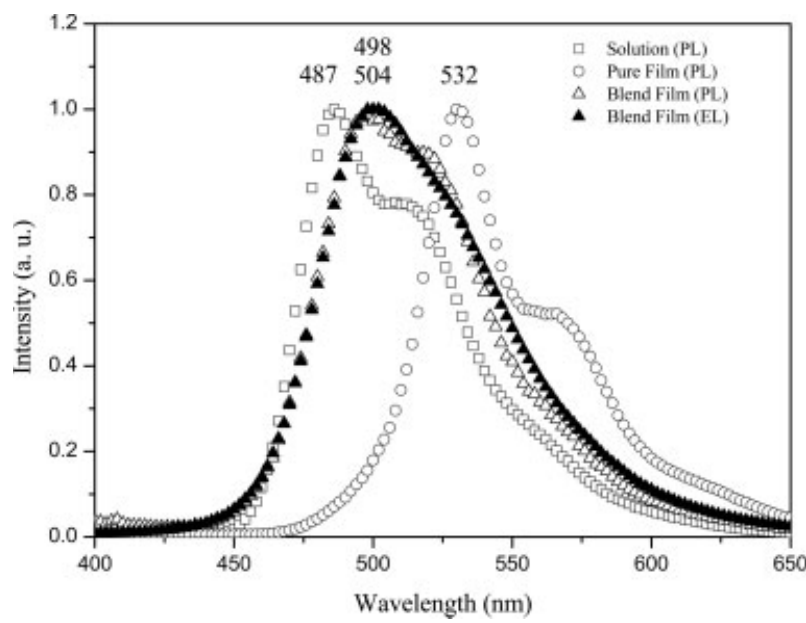

Figure 5. PL and EL spectra of $\mathbf{1 1}$ (the PVK matrix was used in the blend films; chromophore/PVK $=1: 100 \mathrm{wt} \%)$. 


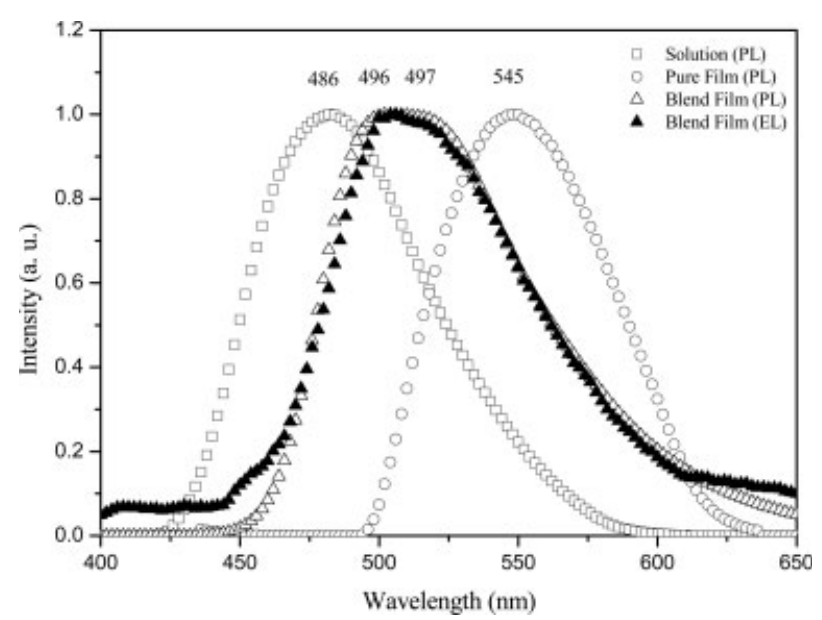

Figure 6. PL and EL spectra of $\mathbf{1 6}$ (the PVK matrix was used in the blend films; chromophore/PVK $=1: 100 \mathrm{wt} \%)$.

Because of the poor film quality of these chromophores, OPVs 1-14 and poly(p-phenylenevinylene)s 15-18 were doped into PVK to form various blend films in a PVK matrix (chromophore/PVK $=1: 100$ wt \%). The EL spectra and $\lambda_{\max }$ values of EL are also shown in Table 5 and Figures 4-6. The current-voltage-luminescence curves of the blend films containing $\mathbf{6 , 1 1}$, and $\mathbf{1 6}$ are shown in Figure 7. Similar turn-on voltages around 9-12 V have been obtained, and the blend film containing three-conjugated ring oligomer 6 has the highest brightness of $120 \mathrm{~cd} / \mathrm{m}^{2}$. Although the highest brightness is only around $120 \mathrm{~cd} / \mathrm{m}^{2}$ for these blend films, they can be further improved to have a brightness greater than $50,000 \mathrm{~cd} / \mathrm{m}^{2}$ by the introduction of hole- and electron-transporting layers in the multilayer design. Related results of various device configurations and characteristics of the multilayer devices will be deliberated in another report.

\section{CONCLUSIONS}

Mesomorphism has been introduced into the BV series by the replacement of three-conjugated rings with five-conjugated phenyl cores, and $\mathbf{1 1}$ has the most appropriate chain length on both terminals (on both end rings) and sides (on the central ring) of the conjugated core and thus has the widest mesophasic range $\left(105{ }^{\circ} \mathrm{C}\right.$ on heating) in the BV series. In comparison with three-conjugated ring BIII oligomers, five-conjugated ring derivatives (BV oligomers and $\mathrm{PBV}$ polymers) have larger $\lambda_{\max }$ values, that is, redshifted absorption, because of the smaller energy gaps of longer conjugations in five-conjugated ring systems. Analogous polymers, that is, the PBV series, containing various side groups have wider mesophasic temperature ranges than those of analogous oligomers because the rigid cores of the polymer backbones are connected by the flexible alkoxy spacers, which enhance the mesogenic longitudinal interaction in the nematic phase. According to the $\lambda_{\max }$ values of PL emissions in various phases (i.e., $\lambda_{\max }$ in the crystalline state $>\lambda_{\max }$ in the LC state $>\lambda_{\max }$ in the isotropic state), the blueshifts of PL emissions may be due to the reduction of coplanar configurations and conjugation lengths via heating to the less ordered phases. In addition, the polarization ratios of PL emissions in various phases reach the maximum values at temperatures just entering the nematic phase, and the PL polarization ratios are reduced upon heating; this is similar to the reduction of the order parameter upon the heating of the LC phase. By the adjustment of flexible alkoxy groups either on the central rings or on both end rings of the conjugated cores, not only the solubility and thermal properties (including the phase behavior and phase-transition temperatures, i.e., $T_{\mathrm{m}}$ and $T_{\mathrm{i}}$ ) but also the PL and EL properties, including the $\lambda_{\max }$ (LUMO, HOMO, fwhm, and energy gap) values, turn-on voltage, brightness, and quantum yield, of both three-conjugated and five-conjugated ring derivatives can be modified easily.

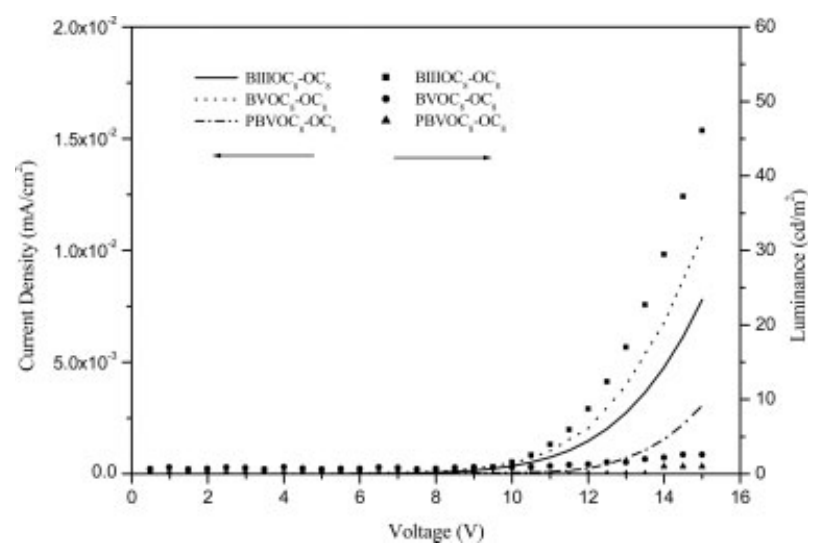

Figure 7. Current-voltage-luminescence curves of blend films containing $\mathbf{6}, \mathbf{1 1}$, and $\mathbf{1 6}$ in the PVK matrix (chromophore/PVK $=1: 100 \mathrm{wt} \%$ ). The lines (solid, dotted, and dashed-dotted) are current-voltage curves, and the shapes (squares, circles, and triangles) are luminescence-voltage curves. 
The authors are grateful for the financial support provided by the National Science Council of Taiwan (Republic of China) through NSC 92-2113-M-009-016 and for the instrumental support provided by Yu-Tai Tao at the Institute of Chemistry of Academia Sinica (Taiwan).

\section{REFERENCES AND NOTES}

1. Sirringhaus, H.; Wilson, R. J.; Friend, R. H.; Inbasekaran, M.; Wu, W.; Woo, E. P.; Grell, M.; Bradley, D. D. C. Appl Phys Lett 2000, 77, 406.

2. West, J. L.; Magyar, G. R.; Kelly, J. R. Appl Phys Lett 1995, 67, 155.

3. Lam, J. W. Y.; Tang, B. Z. J Polym Sci Part A: Polym Chem 2003, 41, 2607.

4. Lam, J. W. Y.; Kong, X.; Dong, Y.; Cheuk, K. K. L.; Xu, K.; Tang, B. Z. Macromolecules 2000, 33, 5027.

5. Chen, J.; Xie, Z.; Lam, J. W. Y.; Law, C. C. W.; Tang, B. Z. Macromolecules 2003, 36, 1108.

6. Huang, Y. M.; Lam, J. W. Y.; Cheuk, K. K. L.; Ge, W.; Tang, B. Z. Mater Sci Eng B 2001, 85, 122.

7. (a) Sung, H. H.; Lin, H. C. Macromolecules 2004, 37, 7945; (b) Sung, H. H.; Lin, H. C. J Polym Sci Part A: Polym Chem 2005, 43, 2700; (c) Lin, H. C.; Sung, H. H.; Tsai, C. M.; Li, K. J. Polymer 2005, 46, 9810.

8. Meisel, A.; Miteva, T.; Glaser, G.; Scheumann, V.; Neher, D. Polymer 2002, 43, 5235.

9. Neher, D. Macromol Rapid Commun 2001, 22, 1365.

10. Banach, M. J.; Friend, R. H.; Sirringhaus, H. Macromolecules 2003, 36, 2838.

11. Millaruelo, M.; Oriol, L.; Pinol, M.; Saez, P. L.; Serrano, J. L. J Photochem Photobiol A 2003, 55, 29.

12. Yang, X. H.; Neher, D.; Spitz, C.; Zojer, E.; Brédas, J. L.; Guntner, R.; Scherf, U. J Chem Phys 2003, 119, 6832 .

13. Godbert, N.; Burn, P. L.; Gilmour, S.; Markham, J. P. J.; Samuel, I. D. W. Appl Phys Lett 2003, 83, 5347.

14. Zen, A.; Neher, D.; Bauer, C.; Asawapirom, U.; Scherf, U.; Hagen, R.; Kosttomine, S.; Mahrt, R. F. Appl Phys Lett 2002, 80, 4699.

15. Whitehead, K. S.; Grell, M.; Bradley, D. D. C.; Jandke, M.; Strohriegel, P. Appl Phys Lett 2000, 76, 2946.

16. Miteva, T.; Meisel, A.; Knoll, W.; Nothofer, H. G.; Scherf, U.; Müller, D. C.; Meerholz, K.; Yasuda, A.; Neher, D. Adv Mater 2001, 13, 565.

17. Grozema, F. C.; Savenije, T. J.; Vermeulen, M. J. W.; Siebbeles, L. D. A.; Warman, J. M.; Meisel, A.; Neher, D.; Nothofer, H. G.; Scherf, U. Adv Mater 2001, 13, 1627.

18. Sainova, D.; Zen, A.; Nothofer, H. G.; Asawapirom, U.; Scherf, U.; Hagen, R.; Bieringer, T.; Kostromine, S.; Neher, D. Adv Funct Mater 2002, 12,49 .
19. Geng, Y.; Culligan, S. W.; Trajkovska, A.; Wallace, J. U.; Chen, S. H. Chem Mater 2003, 15, 542.

20. Geng, Y.; Chen, A. C. A.; Ou, J. J.; Chen, S. H.; Klubek, K.; Vaeth, K. M.; Tang, C. W. Chem Mater 2003, 15, 4352.

21. Hulvat, J. F.; Stupp, S. I. Adv Mater 2004, 16, 589.

22. Chen, J.; Peng, H.; Law, C. C. W.; Dong, Y.; Lam, J. W. Y.; Williams, I. D.; Tang, B. Z. Macromolecules 2003, 36, 4319.

23. Mochizuki, H.; Hasui, T.; Kawamoto, M.; Ikeda, T.; Adachi, C.; Tanighchi, Y.; Shirota, Y. Macromolecules 2003, 36, 3457.

24. Kawamoto, M.; Mochizuki, H.; Shishido, A.; Tsutsumi, O.; Ikeda, T.; Lee, B.; Shirota, Y. Macromolecules 2003, 107, 4887.

25. Chang, S. W.; Li, A. K.; Liao, C. W.; Hsu, C. S. Jpn J Appl Phys 2001, 41, 1374.

26. Grahchev, I.; Moneva, I.; Bojinov, V.; Guittonneau, S. J Mater Chem 2000, 10, 1291.

27. Contoret, A. E. A.; Farrar, S. R.; Jackson, P. O.; O’Neill, M.; Nicholls, J. E.; Kelly, S. M.; Rochards, G. J. Synth Met 2001, 121, 1645.

28. Sung, H. H.; Lin, H. C. Liq Cryst 2004, 31, 831.

29. Pei, Q.; Yang, Y. Adv Mater 1995, 7, 559.

30. Andrew, A. C.; Chen, S. W.; Culligan, Y. G.; Chen, S. H.; Klubek, K. P.; Vaeth, K. M.; Tang, C. W. Adv Mater 2004, 16, 783.

31. Hoag, B. P.; Gin, D. L. Liq Cryst 2004, 31, 185.

32. Precup-Blaga, F. S.; Schenning, A. P. H. J.; Meijer, E. W. Macromolecules 2003, 36, 565.

33. Eckert, J. F.; Nicoud, J. F.; Nierengarten, J. F.; Liu, S. G.; Echegoyen, L.; Barigelletti, F.; Armaroli, N.; Ouali, L.; Krasnikov, V.; Hadziioannou, G. J Am Chem Soc 2000, 122, 7467.

34. Beckers, E. H. A.; Hal, P. A.; Schenning, A. P. H. J.; El-Ghayoury, A.; Peeters, E.; Rispens, M. T.; Hummelen, J. C.; Meijer, E. W.; Janssen, R. A. J. J Mater Chem 2002, 12, 2054.

35. Peeters, E.; van Hal, P. A.; Knol, J.; Brabec, C. J.; Sariciftci, N. S.; Hummelen, J. C.; Janssen, R. A. J. J Phys Chem B 2000, 104, 10174.

36. Wang, H.; Wang, H. H.; Urban, V. S.; Littrell, K. C.; Thiyagarajan, P.; Yu, L. J Am Chem Soc 2000, $122,6855$.

37. Dudek, S. P.; Sikes, H. D.; Chidsey, C. E. D. J Am Chem Soc 2001, 123, 8033.

38. Seferos, D. S.; Banach, D. A.; Alcantar, N. A.; Israelachvili, J. N.; Bazan, G. C. J Org Chem 2004, 69, 1110.

39. Wong, M. S.; Li, Z. H.; Shek, M. F.; Samoc, M.; Samoc, A.; Barry, L. D. Chem Mater 2002, 14, 2999.

40. Jonkheijm, P.; Hoeben, F. J. M.; Kleppinger, R.; Herrikhuyzen, J.; Schenning, A. P. H. J.; Meijer, E. W. J Am Chem Soc 2003, 125, 15941.

41. Jonkheijm, P.; Fransen, M.; Schenning, A. P. H. J.; Meijer, E. W. J Chem Soc Perkin Trans 2001, 2,1280 . 
42. Gesquiere, A.; Jonkheijm, P.; Schenning, A. P. H. J.; Mena-Osteritz, E.; Bauerle, P.; De Feyter, S.; De Schryver, F. C.; Meijer, E. W. J Mater Chem 2003, 13, 2164.

43. Jorgensen, M.; Krebs, F. C. J Org Chem 2004, 69, 6688.

44. Goodson, T.; Li, W.; Gharavi, A.; Yu, L. Adv Mater 1997, 9, 639 .

45. Bittner, R.; Daubler, T. K.; Neher, D.; Meerholz, K. Adv Mater 1999, 11, 119.

46. Wong, M. S.; Li, Z. H.; Tao, Y.; D’Iorio, M. Chem Mater 2003, 15, 1198.

47. Precup-Blaga, F. S.; Garcia-Martinez, J. C.; Schenning, A. P. H. J.; Meijer, E. W. J Am Chem Soc 2003, 125, 12953.

48. Wong, M. S.; Li, Z. H.; Shek, M. F.; Chow, K. H.; Tao, Y.; D’Iorio, M. J Mater Chem 2000, 10, 1805.

49. Lee, G. Y.; Park, B.; Woo, H. S.; Kim, Y.; Ha, C. S.; Lee, C. M.; Jeong, K.; Ha, J. H.; Kim, Y. R. Solid State Commun 1997, 102, 895.
50. Detert, H.; Schollmeyer, D.; Sugiono, E. Eur J Org Chem 2001, 2927.

51. (a) Gebherdt, V.; Bacher, A.; Thelakkat, M.; Stalmach, U.; Meier, H.; Schmidt, H. W.; Haarer, D. Synth Met 1997, 90, 123; (b) Choong, Y.; Park, Y.; Gao, Y.; Wehrmeister, T.; Mullen, K.; Hsieh, B. R.; Tang, C. W. Appl Phys Lett 1996, 69, 1492.

52. Hulvat, J. F.; Sofos, M.; Tajima, K.; Stupp, S. I. J Am Chem Soc 2005, 127, 366.

53. (a) Gill, R. E.; Hadziioannou, G. Adv Mater 1996, 8, 212; (b) Babudri, F.; Farinola, G. M.; Lopez, L. C.; Martinelli, M. G.; Naso, F. J Org Chem 2001, 66,3878 .

54. Brouwer, H. J.; Krasnikov, V. V.; Hilberer, A.; Hadziioannou, G. Adv Mater 1996, 8, 935.

55. (a) Liu, Y.; Liu, M. S.; Li, X. C.; Jen, A. K. Y. Chem Mater 1998, 10, 3301; (b) Ahn, T.; Jang, M. S.; Shim, H. K.; Hwang, D. H.; Zyung, T. Macromolecules 1999, 32, 3279.

56. Peng, Z.; Gharavi, A. R.; Yu, L. J Am Chem Soc 1997, 119, 4622. 